\title{
Shapify: Pathways to SARS-CoV-2 Frameshifting Pseudoknot
}

\author{
Luke Trinity ( $\square$ Ltrinity@uvic.ca ) \\ University of Victoria \\ lan Wark \\ University of Alberta \\ Lance Lansing \\ University of Victoria \\ Hosna Jabbari \\ University of Victoria \\ Ulrike Stege \\ University of Victoria
}

\section{Research Article}

Keywords: secondary structure, frameshifting, pseudoknot

Posted Date: March 7th, 2022

DOI: https://doi.org/10.21203/rs.3.rs-1370718/v1

License: (c) (1) This work is licensed under a Creative Commons Attribution 4.0 International License. Read Full License 


\section{RESEARCH}

\section{Shapify: Pathways to SARS-CoV-2 Frameshifting Pseudoknot}

Luke Trinity $^{1 *}$, Ian Wark ${ }^{2}$, Lance Lansing ${ }^{1}$, Hosna Jabbari ${ }^{1,3 \dagger}$ and Ulrike Stege ${ }^{1 \dagger}$

\footnotetext{
${ }^{*}$ Correspondence: Ltrinity@uvic.ca

${ }^{1}$ Department of Computer

Science, University of Victoria,

Victoria, BC

Full list of author information is

available at the end of the article

†Senior author
}

\begin{abstract}
Background: Multiple viruses including HIV, MERS-CoV (coronavirus responsible for Middle East Respiratory Syndrome, MERS), SARS-CoV (coronavirus responsible for SARS) and SARS-CoV-2 (coronavirus responsible for COVID-19) use a mechanism known as -1 programmed ribosomal frameshifting ( $-1 \mathrm{PRF})$ to successfully replicate. SARS-CoV-2 possesses a unique RNA pseudoknotted structure that stimulates -1 PRF. Recent experiments identified small molecules as antiviral agents that can bind to the pseudoknot and disrupt its stimulation of -1 PRF. Targeting -1 PRF in SARS-CoV-2 to impair viral replication can improve patients' prognoses. Crucial to developing these successful therapies is modeling the structure of the SARS-CoV-2 -1 PRF pseudoknot. Our goal is to expand knowledge of possible pseudoknot conformations.
\end{abstract}

Results: Following a structural alignment approach, we identify similarities in -1 PRF pseudoknots of SARS-CoV-2, SARS-CoV, and MERS-CoV. We introduce Shapify, a novel algorithm that given an RNA sequence incorporates structural reactivity (SHAPE) data and partial structure information to output an RNA secondary structure prediction within a biologically sound hierarchical folding approach. Shapify helps us to better understand non-native SARS-CoV-2 -1 PRF pseudoknot conformations that are relevant to structure function and may correlate with -1 PRF efficiency. We provide in-depth analysis by investigating the structural landscape for the SARS-CoV-2 -1 PRF pseudoknot, including reference and mutated sequences. To better understand the impact of mutations, we provide insight on SARS-CoV-2 -1 PRF pseudoknot sequence mutations and their effect on the resulting structure.

Conclusion: We identify the consensus structure for SARS-CoV, SARS-CoV-2, and MERS-CoV -1 PRF pseudoknots; this similarity in functional RNA structures aids treatment preparation for existing and emergent viruses. Shapify predictions are guided both by SHAPE data and partial structure information. Applied to the SARS-CoV-2 -1 PRF pseudoknot, Shapify unveiled previously unknown pathways from initial stems to pseudoknotted secondary structures. Where SHAPE data is unavailable we provide predictions for noteworthy SARS-CoV-2 -1 PRF mutated pseudoknot sequences. By contextualizing our work with available experimental data, our structure predictions motivate future RNA structure-function research and can aid 3-D modeling of pseudoknots.

Keywords: secondary structure; frameshifting; pseudoknot

\section{Introduction}

4 Sequence analyses of the SARS-CoV-2 genome classify it as a member of the Betacoronavirus subfamily, which includes SARS-CoV and MERS-CoV [1, 2]. Despite having one of the lowest fatality rates among illnesses caused by Betacoronaviruses 
(MERS: 34.3\% [3]; SARS: 11\% [4]; COVID-19: 2.3\% [5]), SARS-CoV-2 caused a pandemic as it is highly contagious. In order to combat this pandemic, and prepare for novel variants or emerging viruses, it is essential to develop treatments for those who become affected.

All coronaviruses use a particular replication strategy called ribosomal frameshifting, which is a promising target for therapeutic drug development of all illnesses caused by coronaviruses [6]. In the case of SARS-CoV-2, two different long open reading frames comprise two-thirds of the viral genome: ORF1a and ORF1b (cf. Figure 1) [7]. ORF1b is out of frame with respect to ORF1a, meaning the ribosome will not translate both frames without shifting from one frame to the other. All coronaviruses utilize the combination of a slippery sequence (where the ribosome is prone to slipping forwards or backwards into a different reading frame) and an $R N A$ pseudoknot to cause the ribosome to shift from one reading frame into the other $[8,9]$.

Usually during translation, the ribosome unwinds the pseudoknot structure and continues translation without shifting reading frames. Sometimes, the pseudoknot causes the ribosome to pause at the slippery sequence, and then shift backwards one nucleotide (referred to as ' -1 ') before continuing in the second reading frame. This molecular mechanism is called ribosomal frameshifting. The -1 shift results in the creation of a fusion ORF1a/1b polypeptide that is vital for viral replication [10]. The site for -1 PRF has great promise as a therapeutic target, because decreasing the rate of ribosomal frameshift (-1 PRF efficiency) can inhibit viral replication [11, 12].

The pseudoknot causing ribosomal frameshifting is an example of RNA secondary structure. When RNA folds back on itself, complementary bases pair, resulting in formation of secondary structure. The most common RNA secondary structure is the hairpin loop. A hairpin loop occurs when RNA folds to form a double helix called a stem, with the unpaired nucleotides forming a single stranded region called a loop (cf. Figure 2a).

The pairing of unpaired bases of a hairpin loop with complementary bases downstream, results in an H-type pseudoknot (cf. Figure 2). Pseudoknotted base pairs are referred to as crossing base pairs, as when viewed in a conventional arc diagram, the bases cross one another (cf. Figure 2b). As the ribosome moves along the RNA during elongation, the pseudoknotted structure represents a kinetic barrier and must be disrupted to allow the next codon into the decoding center (a.k.a. translocation) [13].

RNA folding refers to the process by which RNA acquires secondary structure through interacting nucleotide bases. RNA folding can be described sequentially, with the initial stem forming first (Figure 2, red lines) followed by an additional stem (Figure 2, blue lines). This concept is based on the hierarchical folding hypothesis. This hypothesis posits that RNA first folds into a pseudoknot-free structure, one with no crossing base pairs. Then, additional bases pair that may form pseudoknots to lower the minimum free energy of the structure [14]. The initial structure may be modified locally to accommodate addition of more stable base pairs [15, 16]. The hierarchical folding hypothesis is supported by experts [17, 18, 19]. In particular, experiments on an H-type frameshifting pseudoknot resulted in Stem 1 forming first, followed by the second pseudoknotted stem [20]. 
The expected secondary structures of -1 PRF stimulating pseudoknots, referred to as native or wild-type pseudoknots, have been identified and studied for multiple viruses $[21,22]$. In addition, it has been identified that some non-native pseudoknot conformations, those that differ from the native structure, play a role in regulating frameshifting [21]. Specifically, the conformational plasticity of pseudoknots, i.e., their ability to fold into non-native structures, was found to be correlated with -1 PRF efficiency [21]. Potential non-native secondary structures for the SARSCoV-2 -1 PRF pseudoknot have not been accounted for in recent analyses [23, 24]. Specifically, Omar et al. used the native secondary structure of the SARS-CoV$2-1$ PRF pseudoknot as a starting point in their 3-D physics-based structure prediction [24]. Given that non-native folding pathways correlate with -1 PRF efficiency and are relevant to structure function, further research detailing nonnative SARS-CoV-2 -1 PRF pseudoknot conformations is valuable in structural prediction.

In addition, mutations at various positions have been reported in all components of the SARS-CoV-2 -1 PRF pseudoknot [25, 26]. Yet, the possible effects of such mutations on RNA structure formation and resulting secondary structures have not been fully characterized. Understanding the impact of mutations is vital to long-term success of treatments that are based on predicted RNA structures. Interestingly, the most prevalent mutation to the SARS-CoV-2 - 1 PRF pseudoknot sequence increased its similarity to the MERS-CoV -1 PRF pseudoknot [25]. Further validation of this similarity between the SARS-CoV-2 and MERS-CoV -1 PRF pseudoknots is highly relevant for future coronavirus structure-function prediction efforts.

It was noted that reducing frameshifting efficiency of SARS-CoV attenuated viral propagation to a significant degree [11, 12, 27]. Small molecule therapy was also shown to be effective for SARS-CoV-2 in vitro, where introducing a specific molecule to limit the conformational plasticity of the pseudoknot reduced -1 PRF by $60 \%$ [23]. In addition, the small molecule binding was found to be resilient to mutations in the SARS-CoV-2 -1 PRF sequence [26]. These experimental results demonstrate the potential for SARS-CoV-2 treatments based on -1 PRF inhibition. Increasing comprehensive knowledge of predicted SARS-CoV-2 - 1 PRF pseudoknot structures is valuable in validating small molecule therapeutics.

In this article we identify structural similarities between the -1 PRF pseudoknots of SARS-CoV, SARS-CoV-2, and MERS-CoV. In order to follow up on the hypothesized link between the SARS-CoV-2 and MERS-CoV -1 PRF pseudoknot structures [25], we enumerate the specific loci of similarity as well as a consensus structure. Following the hierarchical folding hypothesis (see Section 2), we present structures for SARS-CoV-2 and MERS-CoV -1 PRF pseudoknots (see Section 3). We developed an algorithm, Shapify, to provide new insight to the SARS-CoV-2 -1 PRF pseudoknot structure, utilizing a hierarchical folding model in combination with three structural reactivity probing experimental datasets as constraints [28, 29, 30, 31]. In the absence of SHAPE information or expert knowledge, we provide pseudoknot predictions for seven SARS-CoV-2 - 1 PRF mutated pseudoknot sequences (see Section 3). These mutations were selected because they have been observed in the population [32] as well as experimentally validated for 
their effect on -1 PRF frameshifting [26]. We discuss the relationship between each set of predicted initial stems and pseudoknotted structures, and provide analysis of the structural reactivity based predictions (see Section 4). Finally, we contextualize our predictions with available experimental work/data including crystallography and cryo-electron microscopy [33, 34].

Whereas previous work recognized the importance of non-native pseudoknotted structures, our research goes further by explicitly identifying non-native structures and predicting how the structure is realized. To the best of our knowledge there is currently no computational method available for prediction of complex suboptimal pseudoknotted secondary structures with SHAPE data. Our results contribute to RNA structural prediction by providing a sampling of the landscape of notable but previously unidentified non-native secondary structures, which may play a role in regulating frameshifting $[21,35]$. Our pseudoknot structural predictions represent alternate starting points which can improve the accuracy of existing 3-D physicsbased modeling [36, 37, 38, 39, 40, 41]. By analyzing predictions based on structural reactivity data, we provide novel information regarding the application of such methods in this specific context. The structural landscape delineated here links initial stems with additional stems that are likely to follow. This approach is unique in providing information about possible pathways to the final pseudoknotted structure. Furthermore, identifying the effect of mutations on structure can be beneficial in designing high efficacy therapies.

\subsection{Background}

It has been experimentally shown that the conformational plasticity of pseudoknots is correlated with $-1 \mathrm{PRF}$ efficiency [21]. One explanation is that ribosomal complex state transitions are modulated by pseudoknot unwinding [22]. It is predicted that pseudoknots with higher plasticity are likely to fold into alternative stable structures that can produce a higher energy barrier in late stage translocation, which in turn initiates translation in the -1 frame [42]. This aligns with the previously held hypothesis that the unwinding pseudoknots may regulate -1 PRF by changing the tension between RNA and ribosomes [43, 44]. The formation of triple-stranded helices has been proposed to contribute to conformational plasticity by enhancing the unwinding resistance of a pseudoknot to the translocating ribosome, contributing to conformational rearrangement [20].

The native structure of the frameshift stimulating pseudoknot in SARS-CoV-2 possesses a unique three-stemmed structure, an H-type pseudoknot (cf. Figure 3). Kelly et al. [23] observed the rate of frameshifting in SARS-CoV-2 to be approximately $15 \%-30 \%$, indicating there is further variation in structure beyond what the authors could capture in their experimental procedure. Indeed, unfolding experiments to investigate structural dynamics of the SARS-CoV-2 frameshifting pseudoknot revealed multiple distinct conformations [45].

Distinct small molecules have been identified, which can bind with the SARSCoV -1 PRF pseudoknot and disrupt its function [46, 47, 48]. Ritchie et al. in 2014 observed that the introduction of the small molecular ligand 2-\{[4-(2-methylthiazol4-ylmethyl)-[1,4]diazepane-1-carbonyl]amino\}benzoic acid ethyl ester (MTDB), effectively decreased alternate folding by binding with the SARS-CoV -1 PRF pseu- 
doknot [47]. Based on their experiment it was predicted that MTDB formed hydrogen bonds with the nucleotides in Loop 3, inhibiting a non-native folding pathway. This binding resulted in reduction of the $-1 \mathrm{PRF}$ rate to near $0 \%$. In vitro experiments for SARS-CoV-2 determined that MTDB binding reduced -1 PRF by $60 \%$ [23]. This result was replicated and expanded upon by experiments that found various mutations in the SARS-CoV-2 - 1 PRF pseudoknot sequence did not have a significant effect on anti-frameshifting activity of MTDB [26]. More recently, the compound merafloxacin was identified as significantly decreasing the efficiency of the -1 frameshift in SARS-CoV-2 [48]. This result was further validated by additional experiments in vivo [49], confirming the viability of targeting the frameshift element as a high-efficacy treatment. The latest frameshift inhibiting novel compound discovered at the time of this writing, 2-(5-acetylthiophen-2yl)furo[2,3-b]quinoline (KCB261770), was found to reduce frameshift efficiency in SARS-CoV, SARS-CoV2, and MERS-CoV [50]. This points to structural similarity between the three coronaviruses, and makes KCB261770 a promising potential therapeutic for a range of coronaviruses.

Mutations have been reported in nearly every position in the SARS-CoV-2 -1 PRF pseudoknot sequences [26] available on GenBank [51] and GISAID [52]. Kelly et al. [23] conducted experiments on SARS-CoV-2 mutants, utilizing 14 mutations to disrupt -1 PRF function. Our analysis is different as we look at single nucleotide mutations that they did not consider, based on those seen in the general population. A single nucleotide change from cytosine to uracil at position $62(\mathrm{C} 62 \mathrm{U})$ was identified in a significant proportion of sequences [25]. Utilizing the COVID-19 CG database [32], which contains over 6.1 million sequences via GISAID [52] as of January 2022, we identify $\mathrm{C} 62 \mathrm{U}$ continues to be the most prevalent mutation in the -1 PRF pseudoknot sequence ( $0.49 \%$ of global sequences). Only two other mutations in the -1 PRF pseudoknot sequence were detected in over $0.1 \%$ of global samples: $\mathrm{A} 8 \mathrm{G}$ in $0.39 \%$, and $\mathrm{C} 43 \mathrm{U}$ in $0.19 \%$ of all sequences. There have not yet been any mutations characteristically detected in the -1 PRF pseudoknot sequences of the B.1.1.7 lineage (a.k.a. 20I/501Y.V1 Variant of Concern), the B.1.351 lineage (a.k.a. 501.V2 variant, 20C/501Y.V2), the B.1.617.2 lineage (a.k.a. Variant of Concern Delta G/478K.V1), or any of the B.1.1.52A+BA.* lineage (a.k.a. Variant of Concern Omicron GRA) or sublineages (B.A.2 and B.A.3 [53]) see Supplementary Materials for accession IDs.

Even a single nucleotide change in an RNA sequence can affect the resulting structure, with implications for function associated with the structure. There is evidence that as little as one or two mutations or deletions can completely disrupt the native structure formation of the SARS-CoV-2 frameshifting pseudoknot [49, 54]. Indeed, Neupane et al. [26] identified a uracil to cytosine mutation at position 20 (U20C) for the SARS-CoV-2 -1 PRF pseudoknot that resulted in more than a three-fold reduction in $-1 \mathrm{PRF}$ efficiency [26]. We further explore the mutations presented in the work of Neupane et al. [26], as these mutations occur in regions important for the formation of the -1 PRF pseudoknot. Three of these mutations are located near the junction of stems (U20C, G29U, U58C), one is adjacent to an adenine bulge identified as critical in frameshifting [27] (C62U), and two are in Loop 2 where mutations have been shown to reduce frameshifting efficiency (C43U, U47C) [55]. 
Incorporating known mutations in pseudoknot structure prediction will advance the understanding of potential conformations which could alter frameshifting efficiency with implications for viral infectivity and pathology.

SHAPE-MaP [56] (selective 2'-hydroxyl acylation analyzed by primer extension and mutational profiling) combines chemical probing and an energy model to identify structural motifs on entire viral RNA genomes [28]. High-throughput structure probing methods including SHAPE-MaP and more recently DMS-MaPseq [57] (dimethyl sulfate (DMS) mutational profiling with sequencing) measure many individual molecules and average the results to generate single-base resolution reactivity data. Recent studies have performed SHAPE-MaP analysis of SARS-CoV-2 in living infected cells [29, 30, 31]. Despite improvements, these high-throughput methods targeting the entire $\sim 30,000 \mathrm{bp}$ genome are prone to noise and may suffer in accurately predicting the structure for a specific region less than 100bp. Here we present structures predicted with and without SHAPE data to better characterize the frameshifting structure.

In addition to secondary structure, the 3-D conformation (e.g. triple-stranded helices) of RNA structures is critical to their function [58]. Most of the existing software packages to determine 3-D conformations of RNA utilize secondary structure as an input constraint [36, 37, 38, 39, 40, 41]. Indeed, the latest 3-D physics-based modeling of the SARS-CoV-2 -1 PRF pseudoknot used the known native secondary structure as a starting point in identifying three unique stable conformations [24]. However, the non-native secondary structures of the SARS-CoV-2 -1 PRF pseudoknot have yet to be identified and incorporated into structural prediction efforts. Our results provide alternate starting points and input constraints, which can improve both the accuracy and interpretability of 3-D physics-based modeling of the pseudoknot.

\section{Methods}

We first present our date sources and availability. Next we introduce methods for structural similarity identification, secondary structure prediction, and the Shapify algorithm. Last, we introduce our procedure for SHAPE data analysis.

\subsection{Data}

The SARS-CoV reference genome NC_004718.3, SARS-CoV-2 reference genome NC_045512.2, and MERS-CoV reference genome NC_019843.3 were obtained from the National Center for Biotechnology Information (NCBI) [59]. For the SARS-CoV reference genome the 68 bp frameshifting pseudoknot sequence occurs at location 13405-13472 [23]. For the SARS-CoV-2 reference genome the 68 bp frameshifting pseudoknot sequence occurs at location 13475-13542 [23]. For the MERS-CoV reference genome the $71 \mathrm{bp}$ frameshifting pseudoknot sequence occurs at location 13440-13510 [25]. The datasets generated and/or analysed during the current study are available in the frameshifting repository: github.com/HosnaJabbari/ frameshifting.

\subsection{Structural Similarity Method:}

RNAz [60], a program for prediction of energetically stable and structurally conserved RNA structures using an alignment based approach, was used to predict 
structural similarity. SARS-CoV-2, SARS-CoV, and MERS-CoV were aligned using Clustal Omega [61] version 1.2.4, with SARS-CoV-2 as the reference.

\subsection{Secondary Structure Prediction}

First we employed a pipeline (cf. Supplementary Materials Figure 9 i.e. Figure S9) with the goal of identifying an array of potential secondary structure conformations, both native and non-native, given an RNA sequence as input. We leveraged the hierarchical folding hypothesis by first predicting a set of energetically favourable initial stems for the input sequence according to the energy parameter set by Andronescu et al. as implemented in HotKnots V2.0 [62, 63]. These energetically favourable initial stems are thermodynamic based, with no SHAPE data adjustment as this was not an option within the HotKnots package. As stated in the hierarchical folding hypothesis, these initial stems are expected to fold first, followed by additional stems [20]. The stems were given unique identifiers and sorted by free energy as calculated using the computeEnergy function from the HotKnots package [62, 63]. While the initial stems have a significant impact on shaping the RNA structure, there is evidence that the original base pairs may be modified locally to accommodate addition of more stable base pair [15, 16]. Therefore, the Iterative HFold algorithm was selected to predict secondary structures [64], which allows the use of an initial predicted stem as input constraint to guide the pseudoknotted secondary structure prediction. Iterative HFold utilizes four biologically sound methods and minimally modifies the input structure to accommodate more stable base pairings. It then outputs the minimum free energy structure among these four predictions as the final structure. Implementing four different methods allows the identification of suboptimal structures, which are candidate structures with a minimum free energy that is only slightly higher than the free energy of the structure with the minimum among all candidates. Here, we utilized the output of these four methods (as opposed to just the one with the minimum free energy) to get a glimpse of the suboptimal structure landscape (cf. Figure 5).

Each initial candidate stem was used as input structure to Iterative HFold. The secondary structures produced based on each initial stem constraint were ranked by their minimum free energy. Each predicted secondary structure was linked to the initial stem(s) that produced it. We report initial stems and the resulting pseudoknotted structures for eight SARS-CoV-2 -1 PRF stimulating pseudoknot sequences: reference, and seven mutated sequences (cf. Table S7).

\subsection{Shapify Method}

In order to incorporate SHAPE data as a constraint within a hierarchical folding approach, we adapted Iterative HFold [64] to develop the Shapify method. Shapify takes as input: an RNA sequence, a SHAPE dataset, and a pseudoknot-free secondary structure. The output from Shapify is an RNA secondary structure prediction. Having inputs of SHAPE data and a pseudoknot-free structure allows for Shapify pseudoknotted predictions to be guided by known RNA structure information. In order to include the SHAPE reactivity information, nearest-neighbor model parameters [63] were adjusted. We define this adjustment below for each nucleotide at index $i$ in an input RNA sequence, following the approach previously utilized by 
Hajdin et al. in their design of the ShapeKnots algorithm [28]. Here, we introduce a penalty for base pairing that increases with experimentally-derived SHAPE reactivity, $m$, and an intercept that encourages base pairing for nucleotides with low SHAPE reactivity, $b$ :

$$
m(\log i+1)+b
$$

The slope and intercept parameters must be determined empirically [28], thus we aggregated a database of 30 RNAs with known structure and available SHAPE datasets. Data from the original ShapeKnots cross-validation [28] included 5 RNAs with lengths $>300 \mathrm{nt}, 5$ riboswitch RNAs, 4 RNAs with structures that are not well predicted with nearest-neighbor thermodynamic parameters, and 3 RNAs whose structures are likely modulated by protein interaction. We supplemented this with 6 RNAs that had a known structure and available SHAPE data via the RNA Mapping Database [65]: 3 riboregulators involved with translation [66], SARS-CoV-2 3' and 5' UTR regions [67], and a ribonuclease domain of Bacillus subtilis [68].

We implemented a leave-one-out cross validation to determine the optimal values for slope $m$ and intercept $b$, searching over a grid of 29 possible slope values and 21 possible intercept values (cf. Figure 4). For each combination we determined the geometric mean of the sensitivity and positive predictive value (ppv) for each of the RNA. We then averaged all the values excluding one RNA respectively, repeating the procedure for each possible RNA to be excluded and taking an average of averages to calculate the final result. Note that base pairs are considered to be predicted correctly when one of the two indices is different by up to one nucleotide, this helps account for uncertainty and dynamism in RNA structure [69]. The longest RNA sequence of length $530 \mathrm{nt}$ took 25 minutes for Shapify to deliver a prediction utilizing a 2nd Generation Intel@ Xeon@ 8171M 2.1GHz (Skylake) with 4 GB memory. We concluded that the optimal values for Shapify parameterization are slope $m=1.4$ and intercept $b=-0.5$ (cf. Figure 4 ), which aligns well with the parameterization for ShapeKnots where $m=1.8$ and $b=-0.6$ were optimal. Shapify is available at github.com/ltrinity/Shapify (DOI: 10.5281/zenodo.6100185).

\subsection{SHAPE data}

We obtained three available SHAPE datasets, one by and referred to as Huston et al. [30], one by and referred to as Manfredonia et al. [29], and another by and referred to as Yang et al. [31]. For each dataset, the values corresponding with the $68 \mathrm{bp}-1$ PRF sequence were used as constraints in RNA secondary structure prediction via the ShapeKnots algorithm [28]. ShapeKnots is a heuristic method based on free energy minimization, that follows the HotKnots [62] approach. The ShapeKnots predictions for each of the three SHAPE datasets can be seen in Table 7.

To further explore the degree to which the information in each SHAPE dataset was used in the structure prediction of ShapeKnots, we implemented a bootstrap method as follows to generate additional datasets based on each original dataset, respectively. For each of the 68 values that could be used as constraint in prediction, the value was selected randomly from the SHAPE dataset, with replacement. Then, this bootstrap dataset was used as constraint for ShapeKnots secondary structure 
prediction. This process was repeated 10,000 times for each of the three SHAPE datasets. Having generated 10,000 secondary structure predictions for each dataset, the secondary structure was converted into binary, with 0 representing unpaired and 1 representing paired. We present both the original SHAPE data (cf. Figure 8a) values as well as the mean and variance of the 10,000 bootstrapped dataset prediction values for each position in the $68 \mathrm{bp}$ sequence (cf. Figure $8 \mathrm{~b}$ ). In addition, we repeated this same bootstrap procedure using the Shapify algorithm instead of ShapeKnots to obtain predictions, in order to compare results between the two methods (cf. Figure 8c).

\section{Results}

We first describe the identified structural similarity between SARS-CoV, SARSCoV-2, and MERS-CoV. Next, we enumerate the secondary structures of the SARSCoV-2 -1 PRF pseudoknot for reference sequence with and without SHAPE data, and for the seven mutated sequences. We then present the secondary structures predicted for the MERS-CoV -1 PRF pseudoknot. Finally, we compare and visualize SARS-CoV-2 -1 PRF pseudoknot SHAPE-based predictions. Table 1 here.

\subsection{Betacoronavirus -1 PRF Pseudoknot Structural Similarity}

One predicted loci of the structural similarity output from RNAz encompassed the region containing the -1 PRF pseudoknot for SARS-CoV-2. Structural similarity between SARS-CoV-2, SARS-CoV, and MERS-CoV was detected at locations 13439 to 13555,13369 to 13485 , and 13404 to 13523 in their respective aligned genomes (cf. Table 1). The RNA-class probability as calculated by RNAz is 0.90 , indicating that the probability for this being a true alignment is approximately $99 \%$.

\subsection{SARS-CoV-2 -1 PRF Pseudoknot}

Table 2 presents three most stable candidates of initial stems for the SARS-CoV-2 -1 PRF stimulating pseudoknot, as calculated based on the reference sequence. For the complete table see Table S2. Using the 18 candidate initial stems as input constraints, 16 secondary structure predictions for the SARS-CoV-2 -1 PRF pseudoknot reference sequence are obtained using the Iterative HFold algorithm [64]. The 11 most stable structures (based on their free energy) are presented in Table 3, see Table S3 for complete table. In our predictions we use parentheses "()" and square brackets "[]" to demarcate pseudoknotted structures. Each period "." identifies an unpaired base. The first column in Table 3 lists the initial stem ID(s) corresponding with the constraint that resulted in the secondary structure prediction. Note that following our pipeline the same structure can result from different initial candidate stems. The secondary structure with the lowest free energy as predicted by Iterative HFold is $100 \%$ consistent with the native SARS-CoV-2 -1 PRF pseudoknot structure. Tables 2 and 3 here.

We repeated the same procedure for each mutated sequence. The predicted initial stems did change in some cases between the reference sequence and the mutated sequences. Certain stems that were predicted for both the reference sequence and respective mutated sequences had a different calculated free energy based on the mutated sequence. Based on our pipeline and the hierarchical folding hypothesis, 
some stems were found for the reference sequence but not for mutated sequences. In addition, specific mutated sequences led to initial stem predictions that were not identified for the reference sequence. We present initial stems for mutated sequences with an ID based on where they would have been ranked by free energy relative to the reference sequence stems (cf. Table 4, Table S7).

Initial stem predictions for the $\mathrm{C} 13 \mathrm{U}$ mutated sequence destabilized Stems 5 and 7 (their free energy increased). One novel stem was detected, referred to as $13 a$ (cf. Table S7). Initial stem predictions for the U20C mutated sequence destabilized Stem 1, but Stems 9, 10, 12, and 16 stabilized (their free energy decreased). Two novel stems were detected referred to as $5 a$ and $5 b$. Repeating the method for the sequence with the guanine/uracil mutation at position 29 (G29U) did not change the predicted energy of any stems, but four stems were not detected (1, 2, 4 and 14). Five novel stems were detected based on the sequence with the G29U mutation, referred to as $2 a, 3 a, 11 a, 13 b$, and $15 a$.

For the sequence with the cytosine/uracil mutation at position 43 (C43U), Stems 4 and 5 destabilized. Stems 6 and 15 were not detected for C43U, and a novel stem was detected, referred to as $4 a$. Stem predictions for the sequence with the uracil/cytosine mutation at position 47 (U47C) stabilized Stem 13; two novel stems were detected, referred to as $4 b$ and $8 a$. For the sequence with the uracil/cytosine mutation at position 58 (U58C), Stems 2 and 18 stabilized. One novel stem was detected, referred to as $18 a$. Finally, for the sequence with the cytosine/uracil mutation at position $62(\mathrm{C} 62 \mathrm{U})$, Stem 16 was not detected. There was a novel stem identified referred to as $13 c$. Table 4 here.

We used the 18 candidate stems from the reference sequence in addition to novel candidate stems for respective mutated sequences (cf. Table S7) as input constraints to predict secondary structures using the Iterative HFold algorithm [64]. For each of the seven SARS-CoV-2 -1 PRF pseudoknot mutated sequences, $14-19$ secondary structures were predicted. However, the predicted secondary structures are markedly different from those predicted based on the reference sequence. The native structure, the structure with lowest free energy in Table 3, was not predicted for U20C, G29U, or C62U mutated sequences based on any of the initial stems. For example, initial Stems 1 and 3 that result in the native structure based on the reference sequence, did not result in the native structure based on the sequence with the U20C mutation (cf. Table S9). Instead, they join initial Stems 8 and 11 resulting in a non-native structure. Between two and five structure clusters (identical prediction from multiple different initial stems) were identified for each mutated sequence. Predictions based on Loop 2 mutations C43U and U47C were similar, which was expected given that mutations in this area were both previously found to reduce frameshifting efficiency [55].

\subsection{SARS-CoV-2 -1 PRF Pseudoknot with SHAPE}

Using the 18 candidate initial stems for the reference sequence as input constraints, each of the three SHAPE datasets, respectively, were also used as constraint in the Shapify algorithm. 14 secondary structure predictions were obtained with Huston et al. [30] dataset as constraint, 19 predictions were obtained with Yang et al. [31] as constraint, and 19 predictions were obtained with Manfredonia et al. [29] as 
constraint. There was significant overlap between the three sets of predictions, giving a total of 36 secondary structure predictions (cf. Table S15).

In Figures 6 and 7 we visualize the pathways from each of the 18 initial stems to the 20 most energetically favourable predicted secondary structures. Beginning from the left, each initial stem is labeled with their ID (cf. Table 2). There were at least three predictions obtained for each initial stem, because each of the three SHAPE datasets was used, respectively, as constraint in the Shapify algorithm. In some cases, more than three predictions were obtained for a single initial stem, due to suboptimal structure prediction (e.g. Stem 8 in Figure 6 with four pathways). Darker color represents higher agreement between SHAPE data. If the prediction for a stem was consistent for all three SHAPE datasets, this is indicated with a black colored pathway. In other cases, where the prediction for a stem was the same for two of the SHAPE datasets as constraint, the pathway is colored dark grey. In the third case, where the prediction was unique to a specific SHAPE dataset as constraint, this pathway is colored light grey.

Pathways shown in Figures 6 and 7 for each initial stem lead to the native structure, or one of the 19 other structures identified by the Shapify algorithm. All structures other than the native are numbered according to their free energy (e.g. NN1 is the most stable non-native structure, NN2 is the second most stable nonnative, etc.). The initial stem IDs are annotated for each secondary structure and respective SHAPE dataset along with all structures' free energies in Table S15. The native structure and the 9 non-native secondary structures that have only minor differences from the native structure are included in Figure 6. Separately, we visualize 10 energetically favourable secondary structures that are significantly different from the native structure (cf. Figure 7). Differences between the native structure in the first row and other structures below are indicated with bold, brackets show differences in paired bases, and asterisks identify bases that are paired in the native structure but unpaired in the non-native structure.

The native structure was reached via six different initial stems as constraint (cf. Figure 6). Structures that were highly similar to the native structure, referred to as native-adjacent structures, also display significant diversity in the pathways with which they can be achieved. Specifically, non-native structures 1, 2, and 4, (i.e. first, second, and fourth most energetically favourable non-native structures) which are highly similar to the native structure, were each reached via two different initial stems as constraints. This pathway redundancy comes in striking contrast to the array of pathways for secondary structures that significantly differed from the native structure (cf. Figure 7). For significantly non-native structures, there were not multiple pathways to the same structure from different initial stems. Furthermore, there was more agreement between predictions from different SHAPE datasets in native and native-adjacent structures than in pathways to significantly non-native structures. This can be seen in the six black pathways in Figure 6 that indicate a consistent prediction when any of the three SHAPE datasets was used as constraint.

\subsection{MERS-CoV -1 PRF Pseudoknot}

Table 5 presents initial candidate stems for the MERS-CoV -1 PRF stimulating pseudoknot, as calculated based on the reference sequence. For the complete set of 
stems see Table S4. Using the 18 initial candidate stems as input constraints, 15 secondary structure predictions for the MERS-CoV -1 PRF pseudoknot reference sequence are obtained using the Iterative HFold algorithm [64]. These predicted pseudoknotted structures are presented in Table 6 sorted by their free energy, see Table S5 for complete table. Note that multiple structures were reached from more than one starting point (cf. rows $1 \& 2$ in Table 6 ). There is no well established native secondary structure for the MERS-CoV -1 PRF pseudoknot. The secondary structure with the lowest free energy as predicted by Iterative HFold is $93 \%$ consistent with the structure presented by Fourmy and Yoshizawa [25]. Tables 5 and 6 here.

\subsection{SARS-CoV-2 -1 PRF Pseudoknot SHAPE Data Analysis}

Table 7 here. Using each of the SHAPE datasets as constraints for ShapeKnots, the three structure predictions (cf. Table 7) had some similarity although it is difficult to see a clear connection with the SHAPE datasets themselves (cf. Figure 8a). There are multiple instances where predictions do not align with SHAPE data. For example, Huston et al. [30] report high reactivity for positions $8-10$, however, the prediction is paired. Conversely, Yang et al. [31] did not report reactivity for positions $8-10$, but the ShapeKnots prediction for these bases was unpaired. Similarly, positions 12 and 13 which had high reactivity reported from the datasets of Huston et al [30] and Yang et al., were predicted as paired when either dataset was used as constraint. This contrasts with predictions based on the dataset from Manfredonia et al. [29], which did not report high reactivity for those positions, but did lead to a prediction of unpaired when the dataset was used as constraint in ShapeKnots.

Predictions via ShapeKnots for all three datasets also reported nucleotides from positions $14-18$ as paired. However, Yang et al. report high reactivity at position 14, and Huston et al. report high reactivity at position 17.

Nucleotides at positions $49-51$ are predicted as paired based on all SHAPE datasets. Yet, the SHAPE dataset of Manfredonia et al. indicates high reactivity for position 51. Nucleotides at positions $53-59$ are predicted as paired based on all SHAPE datasets, while Huston et al. detected high reactivity for nucleotides at positions 55 and 56 and Yang et al. detected high reactivity for position 59. This is indicative of noise in the SHAPE data.

Predictions based on the SHAPE datasets also differed for positions 40, 41, 46 and 47, which were predicted as unpaired based on the dataset of Huston et al. and Yang et al. and paired based on the datasets of Manfredonia et al. In this case the connection between the SHAPE data and the predictions is much clearer. The SHAPE datasets from Huston et al. and Yang et al. had high reactivity reported in this area, while the Manfredonia et al. dataset did not. Overall, certain bases in the -1 PRF sequence are less likely to be affected by the SHAPE data. This can be seen when bootstrapped predictions are close to 0 or 1 , and leads to multiple instances where the SHAPE data does not align with the associated structure prediction. While ShapeKnots has utility in RNA structure prediction, Shapify further investigates the RNA structural landscape by providing predictions based on energetically favourable initial RNA folding. By allowing SHAPE data and pseudoknot-free structure information to guide predictions, Shapify articulates structure formation pathways in a way ShapeKnots cannot. 
In repeating the bootstrap procedure with the Shapify algorithm, there were significant differences with respect to the predictions via ShapeKnots. To begin with, bootstrap results from Shapify predicted the first and second bases in the sequence to be paired far more frequently than ShapeKnots. This is interesting because despite a tendency to predict these bases as paired, three energetically favourable native-adjacent structures were predicted to have these base(s) unpaired (NN2, NN4, and NN5, cf. Figure 6). In these instances, Shapify is guided by partial structure information to the energetically favourable pseudoknotted structure, which is not an option for ShapeKnots. Furthermore, positions 12, 13, 40, 41, 46, 47, and 59 tended to be predicted as paired via ShapeKnots; however, with Shapify these bases were more likely to be predicted as unpaired. Similarly, ShapeKnots tended to predict loci 61 and 62 as unpaired, while Shapify predictions tended more towards a paired prediction. In general, Shapify appears more resilient to extreme values in the SHAPE data, seen by the relatively lower variance especially when Yang et al. [31] data was used.

\section{Discussion \& Conclusions}

We set out to detect structural similarity between different coronaviruses. Here we aligned viral genomes and used RNAz to identify a region of structural similarity for three Betacoronaviruses: SARS-CoV, SARS-CoV-2, and MERS-CoV. In addition to similarity between SARS-CoV and SARS-CoV-2, we detected structural similarity with MERS-CoV in its -1 PRF pseudoknot region. While this result was recently hypothesized by Fourmy and Yoshizawa [25], to the best of our knowledge so far it has not been experimentally validated. In this work, we add more evidence for this structural similarity based on a replicable method. The alignment produced using RNAz, identified a highly conserved region for the -1 PRF pseudoknot sequence of SARS-CoV-2 and MERS-CoV. This is interesting because there is no sequence similarity between the SARS-CoV-2 and MERS-CoV -1 PRF sequences as detected using the Basic Local Alignment Search Tool [70]. Lu et al. found SARS-CoV-2 genome to be $\sim 50 \%$ homologous to MERS-CoV, as compared with $\sim 79 \%$ homologous to SARS-CoV [71]. Similarities in structure between coronaviruses may contain vital information that can validate proposed explanations for frameshifting. The individual and consensus structures presented here can further inform structure prediction, providing previously unavailable insights to explain pathways of structure formation. Understanding structural similarities between coronoviruses can shed light on their mechanism of function and help with finding effective treatments for existing and emerging contagion.

While existing methods that utilize sequence alignment for structure prediction $[29,30,72,73]$ are valuable in genome wide analysis, they are sensitive to hyperparameters and not well-suited to predict potential structures for shorter RNA sequences. Beyond structural similarity, we identify the array of potential secondary structure conformations for the SARS-CoV-2 and MERS-CoV -1 PRF pseudoknots. Here we employed a hierarchical folding based method for prediction of the secondary structures that can also incorporate SHAPE data as constraint.

By following the hierarchical folding hypothesis we explored the structural landscape of the SARS-CoV-2 and MERS-CoV -1 PRF pseudoknots, expanding on 
previous structure prediction efforts [25,33]. We enumerate possible non-native and suboptimal SARS-CoV-2 -1 PRF pseudoknotted structures for the reference SARS$\mathrm{CoV}-2$ sequence as well as seven mutated sequences. We determined the effects of noteworthy mutations to the SARS-CoV-2 -1 PRF pseudoknot sequence [25], finding that certain mutations may stabilize or destabilize pseudoknot structure. These mutated sequences were recently tested for their effect on -1 PRF efficiency [26]. $\mathrm{C} 13 \mathrm{U}$ and $\mathrm{C} 43 \mathrm{U}$ mutations destabilized the structure, while U20C, U47C, U58C mutations stabilized the structure. U20C was the only single mutation found to decrease -1 PRF efficiency [26], which we hypothesize may have been caused by the highly non-native structure ranked third in terms of free energy. Another possible explanation could be the suboptimal structure detected for initial Stem 1 unique to this mutated sequence, it could show competition for formation of Stem 1 which may be crucial in function. Our results demonstrate that, while the native structure of the pseudoknot has the lowest free energy, there are multiple predicted non-native structures that are comparable both from an energetic and structural standpoint. This is further evidence of the conformational plasticity in the three-stemmed pseudoknot structure.

We introduced a method, Shapify, which takes as input an RNA sequence, SHAPE data, and partial structure information to output an RNA secondary structure prediction based on the hierarchical folding approach. Novel predictions from Shapify disclosed pathways from initial stems to secondary structures that showcase remarkable redundancy in the mechanism for achieving the native SARS-CoV-2 -1 frameshifting pseudoknot secondary structure, as well as native-adjacent structures. The Shapify method supports the use of SHAPE data as a guide, leading to novel structure predictions that capture both the conformational flexibility and diverse structural landscape of the SARS-CoV-2 -1 PRF pseudoknot. In particular, we can now compare predictions when different SHAPE datasets are each used as constraint for the same sequence, providing information that was previously unavailable to identify a more complete structural landscape. Given the differences in the SHAPE datasets themselves, adjustment to secondary structure predictions are a logical result that validates the sensitivity of the Shapify method to different SHAPE data as constraint. We found more consistency in predictions for native and native-adjacent structures: multiple paths from different initial stems to these structures, and agreement when various SHAPE datasets were each used as constraint. The frameshift is needed for successful SARS-CoV-2 viral replication; thus we expect the frameshift inducing structure to have resiliency in its formation. Here we identify this native redundancy and contrast it with the set of pathways to significantly non-native structures that are also energetically favourable. By more comprehensively investigating the energetic structural landscape we can better understand this complex biological mechanism. For future work we seek to use SHAPE data to inform the prediction of the initial stems themselves, which are currently only based on thermodynamic properties.

There were three potential structures highlighted by the analysis of Schlick et al. in a method combining graph theory, secondary structure prediction, SHAPE structural probing, and thermodynamic ensemble modeling [74]. One of the structures predicted by Schlick et al. (denoted '3_6') was highly similar to the first, 
second, and fourth most energetically favourable non-native structures identified by the Shapify method. Our results indicate that these structures share pathways to form following the pairing of initial Stems 3, 8, or 14. In addition, both initial Stems 8 and 14 had pathways to two of the ' $3 \_6$ ' adjacent structures, depending on which SHAPE dataset was used as constraint. In the case of initial Stem 8 leading to the formation of the fourth most energetically favourable non-native structure, the pathway is suboptimal, as initial Stem 8 can also lead to the formation of the first or second most energetically favourable non-native structures. By comparing our results with other findings such as those from Schlick et al. [74] we can better understand nuances of frameshifting stimuli that may be critical in effective treatments.

SHAPE-MaP data provides valuable insight into secondary structure, and will continue to be a valuable tool for researchers. By contrasting predictions with SHAPE data analysis, our holistic method provides insight that motivates future RNA structure-function research. With regard to the impact of SHAPE datasets on prediction of secondary structure via SHAPEKnots, there were discrepancies in multiple positions. SHAPE datasets indicated high reactivity that was not evident in the actual secondary structure prediction. By looking at the bootstrapped ShapeKnots predictions, we found that approximately half of the nucleotides were not impacted at all by randomly sampling from the original dataset with replacement, for Manfredonia et al. and Huston et al., respectively. The Yang et al. dataset showed more variability in bootstrapping, which we can attribute to more extreme values in the dataset. From this sensitivity we infer that additional normalization of SHAPE data may be necessary to properly utilize SHAPEKnots.

By repeating the bootstrap procedure with Shapify, we found that despite more extreme values within the Yang et al. [31] SHAPE dataset, their impact was less severe in the bootstrap predictions via Shapify. This indicates more resiliency in the Shapify method. Furthermore, while ShapeKnots is solely reliant on SHAPE data to guide prediction, Shapify can better capture the conformational flexibility of RNA because it is also guided by partial structure information. This was seen by the prediction of energetically favourable structures that were different than the trend observed during the bootstrapping procedure.

Note that SHAPE data is collected in a probing of the entire structure of the $30,000+$ bp viral genome. Therefore, the data represents a secondary structure that extends beyond the $68 \mathrm{bp}$ frameshifting region we are interested in. If a secondary structure spans across the slippery sequence, the data may fail to provide relevant information about the pseudoknot at this specific location with the ability to initiate frameshifting. This is because as the ribosome moves along the RNA, it will unfold structures in its path before reaching the slippery sequence. SHAPE data may reflect secondary structure that forms between the sequence of interest and upstream bases; however, such structures would not be relevant to frameshifting, which occurs when the ribosome is in place over the slippery sequence. To determine the extent this is occurring, additional structural probing experiments are needed that focus specifically on the -1 PRF site.

The three SHAPE datasets presented here have some similar trends but are unique at various positions. This could be attributed to the different protocols 
or approaches for data collection. It may also indicate that the RNA was in different structure conformations for each individual measurement during the highthroughput experiment. Structural probing methods measure multiple molecules and then average the results to obtain final reactivity data, which may lead to noise in the signal when various molecules are each in different structure conformations. To address this, we propose further validation to confirm the consistency of structural conformation across multiple molecules which may be measured in structural probing experiments.

Zhang et al. [33] used cryo electro microscopy (cryo-EM) to solve the SARS-CoV-2 -1 PRF pseudoknot accurate to 6.7 angstroms $\left(10^{-10}\right.$ meters $)$, their result centered around a ring conformation hypothesis for frameshifting. Of interest is that Zhang et al. identified the same 3D RNA structure in a range of betacoronaviruses including MERS-CoV, SARS-CoV, and SARS-CoV-2. This aligns well with the structural similarity we present, and our results can provide context for the predictions of Zhang et al. [33] and others by linking initial stems to pseudoknotted structure and unveiling how mutations may affect structure prediction. More recent cryo-EM experiments identify a structure that is highly similar to the previously mentioned '3_6', although not identical [49]. Cutting-edge crystallography has now been used to solve the SARS-CoV-2 -1 PRF pseudoknot accurate to 2.09 angstroms [34] and now 1.3 angstroms [75]. While similar to previous cryo-EM imaging of the pseudoknot, the crystallography also unveiled marked differences in structure, including a shorter Stem 2 [34]. By applying the Shapify method here we have also identified a shorter Stem 2 in non-native structures 1, 14, and 15, with the relevant bases instead joining Stem 1 or remaining unpaired. In addition we have shown the pathway to these non-native structures, in this case resulting from initial Stems 3, 8, 14, 16, and 18. Further integration of secondary and tertiary structure prediction will contribute to a more complete structural understanding of pseudoknots. Here we have unveiled initial and intermediate transient structures that may be involved in this complex process. Specific to the SARS-CoV-2 -1 PRF pseudoknot, further investigation is needed to determine the frequency that different non-native secondary structures are realized. Emphasis should be placed on understanding the refolding dynamics of partially unfolded structures, which may mimic the interplay of the ribosome and pseudoknot in -1 PRF.

In addition to native-adjacent structures, we predicted non-native structures that are very different from the native expectation. These non-native structures are energetically close to the native structure and likely play a role in regulating frameshifting. In both SARS-CoV-2 and MERS-CoV, the second most energetically favourable stem (Stem 2, cf. Tables 2 and 5) results in a structure prediction that is very different from the native expectation (cf. Tables 3 and 6). Although Stem 2 is not the most energetically favourable, its formation is supported by our structural alignment. Further experiments are needed to confirm the exact structure of non-native conformations for the SARS-CoV-2 -1 PRF pseudoknot and how these specific structures correlate with frameshifting efficiency.

For SARS-CoV-2 reference sequence predictions, five predicted pseudoknotted structures including the native structure, resulted from multiple initial stem input constraints. These structures can be accessed from different starting points, which 
may indicate their increased conformational plasticity. Our identification of possible pathways by which each pseudoknotted structure is formed advances existing understanding of structure formation.

Secondary structure predictions based on the SARS-CoV-2 -1 PRF pseudoknot mutated sequences are demonstrably different than those for the reference sequence. Interestingly, while there were changes in predicted secondary structures, the function of the pseudoknot is expected to be conserved for all mutations other than U20C [26]. Given that the structure of the pseudoknot with sequence mutations is different but still functional, we hypothesize that the relationship between pseudoknot structure and function may be more dynamic and flexible than previously expected.

In the case of the U20C mutation, we identify a structure that is very different from the native expectation and has a low free energy (cf. Table S9 row 3). This highly non-native structure is ranked third in terms of free energy, which is not the case for any other mutation. In addition, U20C mutation was the only SARS-CoV-2 sequence that led to a suboptimal structure detected for inital Stem 1, the most likely initial stem. Further experiments are needed to validate this result and confirm the structural cause for the decrease in -1 PRF efficiency based on the U20C mutation. As existing and new SARS-CoV-2 mutations become more prevalent, determining their impact on -1 PRF pseudoknot structures is key to long-term success of small molecule therapy.

Finally, RNA tertiary contact prediction tools are poised to play an essential role in developing and vetting small molecule therapies. Previous modeling of the SARS-CoV-2 -1 PRF pseudoknot acknowledged the relevance of non-native structures and conformational plasticity [21], but this concept has not been fully integrated into the latest prediction efforts [24]. Efforts in identifying unique stable $3 \mathrm{D}$ conformations of the SARS-CoV-2 -1 PRF pseudoknot can have a great impact in treatment development [24]. We believe such endeavors can benefit from a more comprehensive overview of the SARS-CoV-2 -1 PRF pseudoknot secondary structure landscape, one that includes non-native structures. Given the complicated nature of modeling tertiary interactions, the initialization of such simulations should account for multiple initial secondary structures of the pseudoknot. Our structural predictions can be utilized in 3-D physics-based modeling of pseudoknots as alternative starting points or to provide context to SARS-CoV-2 structure prediction efforts, where small secondary structure differences can have a profound impact on resulting tertiary interactions and three-dimensional conformations of the pseudoknot $[36,37,38,39,40,41]$.

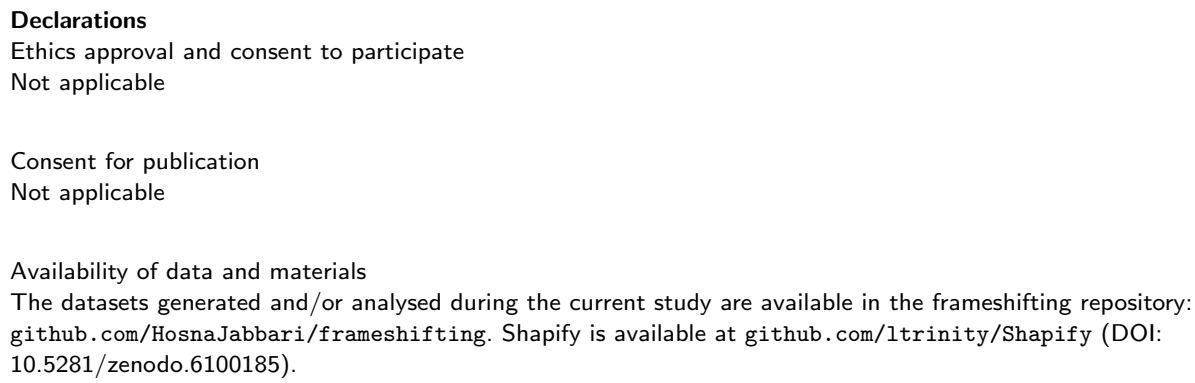


Competing interests

The authors declare that they have no competing interests.

Funding

Funding provided by University of Victoria Department of Computer Science and the Natural Sciences and Engineering Research Council to support the authors; as well as the Microsoft Al4Health Azure Grant enabling data collection via cloud computing.

Author's contributions

LT and HJ designed the research. IW developed the Shapify method. LT and LL carried out all simulations and modeling. LT drafted the manuscript. HJ and US supervised the research. LT, HJ, and US contributed to the revision process.

Acknowledgements

We thank and acknowledge the University of Victoria (UVic), Department of Computer Science for sponsoring this research through the UVic Fellowship Award. We also extend thanks for the Microsoft Al4Health Azure Grant, without which this research would not be possible. In addition, we recognize and express appreciation for the Natural Sciences and Engineering Research Council supporting this work. Finally, we thank the Computational Biology Research and Analytics Lab for invaluable feedback.

\section{Author details}

${ }^{1}$ Department of Computer Science, University of Victoria, Victoria, BC. ${ }^{2}$ Department of Computer Science,

University of Alberta, Edmonton, AB. ${ }^{3}$ Fellow of the Institute on Aging and Lifelong Health, Victoria, BC.

\section{References}

1. Chen, Y., Liu, Q., Guo, D.: Emerging coronaviruses: genome structure, replication, and pathogenesis. J. Med. Virol. 92(4), 418-423 (2020)

2. Petrosillo, N., Viceconte, G., Ergonul, O., Ippolito, G., Petersen, E.: COVID-19, SARS and MERS: are they closely related? Clin. Microbiol. Infect. (2020)

3. Memish, Z.A., Perlman, S., Van Kerkhove, M.D., Zumla, A.: Middle east respiratory syndrome. Lancet (2020)

4. WHO/CDS/CSR/GAR: Consensus document on the epidemiology of severe acute respiratory syndrome (SARS). World Health Organization (2003)

5. Surveillances, V.: The epidemiological characteristics of an outbreak of 2019 novel coronavirus diseases (COVID-19)-China, 2020. China CDC Weekly 2(8), 113-122 (2020)

6. Ghosh, A.K., Brindisi, M., Shahabi, D., Chapman, M.E., Mesecar, A.D.: Drug development and medicinal chemistry efforts toward SARS-Coronavirus and COVID-19 therapeutics. Chem Med Chem (2020)

7. Khailany, R.A., Safdar, M., Ozaslan, M.: Genomic characterization of a novel SARS-CoV-2. Gene reports, $100682(2020)$

8. Dinman, J.D.: Mechanisms and implications of programmed translational frameshifting. Wiley Interdiscip. Rev. RNA 3(5), 661-673 (2012)

9. Atkins, J.F., Loughran, G., Bhatt, P.R., Firth, A.E., Baranov, P.V.: Ribosomal frameshifting and transcriptional slippage: From genetic steganography and cryptography to adventitious use. Nucleic Acids Res. 44(15), 7007-7078 (2016)

10. Fehr, A.R., Perlman, S.: Coronaviruses: an overview of their replication and pathogenesis. Coronaviruses, 1-23 (2015)

11. Plant, E.P., Rakauskaitè, R., Taylor, D.R., Dinman, J.D.: Achieving a golden mean: mechanisms by which coronaviruses ensure synthesis of the correct stoichiometric ratios of viral proteins. J. Virol. 84(9), 4330-4340 (2010)

12. Plant, E.P., Sims, A.C., Baric, R.S., Dinman, J.D., Taylor, D.R.: Altering SARS coronavirus frameshift efficiency affects genomic and subgenomic RNA production. Viruses 5(1), 279-294 (2013)

13. Qu, X., Wen, J.-D., Lancaster, L., Noller, H.F., Bustamante, C., Tinoco, I.: The ribosome uses two active mechanisms to unwind messenger RNA during translation. Nature 475(7354), 118-121 (2011)

14. Tinoco Jr, I., Bustamante, C.: How RNA folds. J. Mol. Biol. 293(2), 271-281 (1999)

15. Wilkinson, K.A., Merino, E.J., Weeks, K.M.: RNA SHAPE chemistry reveals nonhierarchical interactions dominate equilibrium structural transitions in tRNAAsp transcripts. J. Am. Chem. Soc. 127(13), 4659-4667 (2005)

16. Ding, F., Sharma, S., Chalasani, P., Demidov, V.V., Broude, N.E., Dokholyan, N.V.: Ab initio RNA folding by discrete molecular dynamics: from structure prediction to folding mechanisms. RNA 14(6), 1164-1173 (2008)

17. Elstner, M.: The scc-dftb method and its application to biological systems. Theor. Chem. Acc. 116(1), 316-325 (2006)

18. Cho, S.S., Pincus, D.L., Thirumalai, D.: Assembly mechanisms of RNA pseudoknots are determined by the stabilities of constituent secondary structures. PNAS 106(41), 17349-17354 (2009)

19. Bailor, M.H., Sun, X., Al-Hashimi, H.M.: Topology links RNA secondary structure with global conformation, dynamics, and adaptation. Science 327(5962), 202-206 (2010)

20. Chen, G., Chang, K.-Y., Chou, M.-Y., Bustamante, C., Tinoco, I.: Triplex structures in an RNA pseudoknot enhance mechanical stability and increase efficiency of -1 ribosomal frameshifting. PNAS 106(31), 12706-12711 (2009)

21. Ritchie, D.B., Foster, D.A., Woodside, M.T.: Programmed -1 frameshifting efficiency correlates with RNA pseudoknot conformational plasticity, not resistance to mechanical unfolding. PNAS 109(40), 16167-16172 (2012) 
22. Wu, B., Zhang, H., Sun, R., Peng, S., Cooperman, B.S., Goldman, Y.E., Chen, C.: Translocation kinetics and structural dynamics of ribosomes are modulated by the conformational plasticity of downstream pseudoknots. Nucleic Acids Res. 46(18), 9736-9748 (2018)

23. Kelly, J.A., Olson, A.N., Neupane, K., Munshi, S., San Emeterio, J., Pollack, L., Woodside, M.T., Dinman, J.D.: Structural and functional conservation of the programmed -1 ribosomal frameshift signal of SARS coronavirus 2 (SARS-CoV-2). J. Biol. Chem., 120 (2020)

24. Omar, S.I., Zhao, M., Sekar, R.V., Moghadam, S.A., Tuszynski, J.A., Woodside, M.T.: Modeling the structure of the frameshift-stimulatory pseudoknot in SARS-CoV-2 reveals multiple possible conformers. PLoS computational biology 17(1), 1008603 (2021)

25. Fourmy, D., Yoshizawa, S.: A cytosine-to-uracil change within the programmed -1 ribosomal frameshift signal of SARS-CoV-2 results in structural similarities with the MERS-CoV signal. bioRxiv (2020)

26. Neupane, K., Munshi, S., Zhao, M., Ritchie, D.B., Ileperuma, S.M., Woodside, M.T.: Anti-frameshifting ligand active against SARS coronavirus-2 is resistant to natural mutations of the frameshift-stimulatory pseudoknot. J. Mol. Biol. 432(21), 5843-5847 (2020)

27. Plant, E.P., Pérez-Alvarado, G.C., Jacobs, J.L., Mukhopadhyay, B., Hennig, M., Dinman, J.D.: A three-stemmed mRNA pseudoknot in the SARS coronavirus frameshift signal. PLoS Biol. 3(6), 172 (2005)

28. Hajdin, C.E., Bellaousov, S., Huggins, W., Leonard, C.W., Mathews, D.H., Weeks, K.M.: Accurate SHAPE-directed RNA secondary structure modeling, including pseudoknots. PNAS 110(14), 5498-5503 (2013)

29. Manfredonia, I., Nithin, C., Ponce-Salvatierra, A., Ghosh, P., Wirecki, T.K., Marinus, T., Ogando, N.S., Snijder, E.J., van Hemert, M.J., Bujnicki, J.M., et al.: Genome-wide mapping of SARS-CoV-2 rna structures identifies therapeutically-relevant elements. Nucleic Acids Res. 48(22), 12436-12452 (2020)

30. Huston, N.C., Wan, H., Strine, M.S., Tavares, R.d.C.A., Wilen, C.B., Pyle, A.M.: Comprehensive in vivo secondary structure of the SARS-CoV-2 genome reveals novel regulatory motifs and mechanisms. Mol. Cell 81(3), 584-598 (2021)

31. Yang, S.L., DeFalco, L., Anderson, D.E., Zhang, Y., Aw, J.G.A., Lim, S.Y., Lim, X.N., Tan, K.Y., Zhang, T., Chawla, T., et al:: Comprehensive mapping of SARS-CoV-2 interactions in vivo reveals functional virus-host interactions. Nat. Commun. 12(1), 1-15 (2021)

32. Chen, A.T., Altschuler, K., Zhan, S.H., Chan, Y.A., Deverman, B.E.: COVID-19 CG enables SARS-CoV-2 mutation and lineage tracking by locations and dates of interest. Elife 10, 63409 (2021)

33. Zhang, K., Zheludev, I.N., Hagey, R.J., Haslecker, R., Hou, Y.J., Kretsch, R., Pintilie, G.D., Rangan, R., Kladwang, W., Li, S., et al.: Cryo-EM and antisense targeting of the 28-kDa frameshift stimulation element from the SARS-CoV-2 RNA genome. Nat. Struct. Mol. Biol. 28(9), 747-754 (2021)

34. Roman, C., Lewicka, A., Koirala, D., Li, N.-S., Piccirilli, J.A.: The SARS-CoV-2 programmed -1 ribosomal frameshifting element crystal structure solved to 2.09 å using chaperone-assisted RNA crystallography. ACS Chem. Biol. (2021)

35. Trinity, L., Lansing, L., Jabbari, H., Stege, U.: SARS-CoV-2 ribosomal frameshifting pseudoknot: Detection of inter-viral structural similarity. In: 2021 IEEE 9th International Conference on Healthcare Informatics (ICHI), pp. 451-460 (2021)

36. Wang, J., Wang, J., Huang, Y., Xiao, Y.: 3dRNA v2. 0: An updated web server for RNA 3d structure prediction. Int. J. Mol. Sci. 20(17), 4116 (2019)

37. Zok, T., Antczak, M., Zurkowski, M., Popenda, M., Blazewicz, J., Adamiak, R.W., Szachniuk, M.: RNApdbee 2.0: multifunctional tool for RNA structure annotation. Nucleic Acids Res. 46(W1), 30-35 (2018)

38. Li, S., Olson, W.K., Lu, X.-J.: Web 3DNA 2.0 for the analysis, visualization, and modeling of $3 \mathrm{~d}$ nucleic acid structures. Nucleic Acids Res. 47(W1), 26-34 (2019)

39. Biesiada, M., Purzycka, K.J., Szachniuk, M., Blazewicz, J., Adamiak, R.W.: Automated RNA 3d structure prediction with RNAComposer, 199-215 (2016)

40. Parisien, M., Major, F.: The MC-Fold and MC-Sym pipeline infers RNA structure from sequence data. Nature 452(7183), 51-55 (2008)

41. Krokhotin, A., Houlihan, K., Dokholyan, N.V.: iFoldRNA v2: folding RNA with constraints. Bioinformatics 31(17), 2891-2893 (2015)

42. Caliskan, N., Katunin, V.I., Belardinelli, R., Peske, F., Rodnina, M.V.: Programmed -1 frameshifting by kinetic partitioning during impeded translocation. Cell 157(7), 1619-1631 (2014)

43. Plant, E.P., Dinman, J.D.: Torsional restraint: a new twist on frameshifting pseudoknots. Nucleic Acids Res. 33(6), 1825-1833 (2005)

44. Li, Y., Treffers, E.E., Napthine, S., Tas, A., Zhu, L., Sun, Z., Bell, S., Mark, B.L., Van Veelen, P.A., Van Hemert, M.J., et al.: Transactivation of programmed ribosomal frameshifting by a viral protein. PNAS 111(21), 2172-2181 (2014)

45. Neupane, K., Zhao, M., Lyons, A., Munshi, S., Ileperuma, S.M., Ritchie, D.B., Hoffer, N.Q., Narayan, A., Woodside, M.T.: Structural dynamics of single SARS-CoV-2 pseudoknot molecules reveal topologically distinct conformers. Nat. Commun. 12(1), 1-9 (2021)

46. Park, S.-J., Kim, Y.-G., Park, H.-J.: Identification of RNA pseudoknot-binding ligand that inhibits the -1 ribosomal frameshifting of SARS-coronavirus by structure-based virtual screening. J. Am. Chem. Soc. 133(26), 10094-10100 (2011)

47. Ritchie, D.B., Soong, J., Sikkema, W.K., Woodside, M.T.: Anti-frameshifting ligand reduces the conformational plasticity of the SARS virus pseudoknot. J. Am. Chem. Soc. 136(6), 2196-2199 (2014)

48. Sun, Y., Abriola, L., Niederer, R.O., Pedersen, S.F., Alfajaro, M.M., Monteiro, V.S., Wilen, C.B., Ho, Y.-C., Gilbert, W.V., Surovtseva, Y.V., et al.: Restriction of SARS-CoV-2 replication by targeting programmed -1 ribosomal frameshifting. PNAS 118(26) (2021)

49. Bhatt, P.R., Scaiola, A., Loughran, G., Leibundgut, M., Kratzel, A., Meurs, R., Dreos, R., O'Connor, K.M., McMillan, A., Bode, J.W., et al.: Structural basis of ribosomal frameshifting during translation of the SARS-CoV-2 RNA genome. Science 372(6548), 1306-1313 (2021)

50. Ahn, D.-G., Yoon, G.Y., Lee, S., Ku, K.B., Kim, C., Kim, K.-D., Kwon, Y.-C., Kim, G.-W., Kim, B.-T., Kim, 
S.-J.: A novel frameshifting inhibitor having antiviral activity against zoonotic coronaviruses. Viruses 13(8), 1639 (2021)

51. Benson, D.A., Cavanaugh, M., Clark, K., Karsch-Mizrachi, I., Lipman, D.J., Ostell, J., Sayers, E.W.: Genbank. Nucleic Acids Res. 41(D1), 36-42 (2012)

52. Shu, Y., McCauley, J.: Gisaid: Global initiative on sharing all influenza data-from vision to reality. Eurosurveillance 22(13), 30494 (2017)

53. Desingu, P.A., Nagarajan, K., Dhama, K.: Emergence of omicron third lineage BA.3 and its importance. J. Med. Virol. (2022)

54. Schlick, T., Zhu, Q., Jain, S., Yan, S.: Structure-altering mutations of the SARS-CoV-2 frameshifting RNA element. Biophys. J. 120(6), 1040-1053 (2021)

55. Ishimaru, D., Plant, E.P., Sims, A.C., Yount Jr, B.L., Roth, B.M., Eldho, N.V., Perez-Alvarado, G.C., Armbruster, D.W., Baric, R.S., Dinman, J.D., et al.: RNA dimerization plays a role in ribosomal frameshifting of the SARS coronavirus. Nucleic Acids Res. 41(4), 2594-2608 (2013)

56. Siegfried, N.A., Busan, S., Rice, G.M., Nelson, J.A., Weeks, K.M.: RNA motif discovery by SHAPE and mutational profiling (SHAPE-MaP). Nat. Med. 11(9), 959-965 (2014)

57. Zubradt, M., Gupta, P., Persad, S., Lambowitz, A.M., Weissman, J.S., Rouskin, S.: DMS-MaPseq for genome-wide or targeted RNA structure probing in vivo. Nat. Med. 14(1), 75-82 (2017)

58. Butcher, S.E., Pyle, A.M.: The molecular interactions that stabilize RNA tertiary structure: RNA motifs, patterns, and networks. Acc. Chem. Res. 44(12), 1302-1311 (2011)

59. Brister, J.R., Ako-Adjei, D., Bao, Y., Blinkova, O.: NCBI viral genomes resource. Nucleic Acids Res. 43(D1), 571-577 (2015)

60. Gruber, A.R., Findeiß, S., WashietI, S., Hofacker, I.L., Stadler, P.F.: RNAz 2.0: improved noncoding RNA detection. Biocomputing, 69-79 (2010)

61. Sievers, F., Wilm, A., Dineen, D., Gibson, T.J., Karplus, K., Li, W., Lopez, R., McWilliam, H., Remmert, M., Söding, J., Thompson, J.D., Higgins, D.G.: Fast, scalable generation of high-quality protein multiple sequence alignments using clustal omega. Mol. Syst. Biol. 7 (2011)

62. Ren, J., Rastegari, B., Condon, A., Hoos, H.H.: Hotknots: heuristic prediction of RNA secondary structures including pseudoknots. RNA 11(10), 1494-1504 (2005)

63. Andronescu, M.S., Pop, C., Condon, A.E.: Improved free energy parameters for RNA pseudoknotted secondary structure prediction. RNA 16(1), 26-42 (2010)

64. Jabbari, H., Condon, A.: A fast and robust iterative algorithm for prediction of RNA pseudoknotted secondary structures. BMC Bioinform. 15(1), 147 (2014)

65. Cordero, P., Lucks, J.B., Das, R.: An RNA mapping database for curating RNA structure mapping experiments. Bioinformatics 28(22), 3006-3008 (2012)

66. Watters, K.E., Abbott, T.R., Lucks, J.B.: Simultaneous characterization of cellular RNA structure and function with in-cell SHAPE-Seq. Nucleic Acids Res. 44(2), 12-12 (2016)

67. Rangan, R., Watkins, A.M., Chacon, J., Kretsch, R., Kladwang, W., Zheludev, I.N., Townley, J., Rynge, M., Thain, G., Das, R.: De novo 3d models of SARS-CoV-2 RNA elements from consensus experimental secondary structures. Nucleic Acids Res. 49(6), 3092-3108 (2021)

68. Lucks, J.B., Mortimer, S.A., Trapnell, C., Luo, S., Aviran, S., Schroth, G.P., Pachter, L., Doudna, J.A., Arkin, A.P.: Multiplexed RNA structure characterization with selective 2'-hydroxyl acylation analyzed by primer extension sequencing (SHAPE-Seq). PNAS 108(27), 11063-11068 (2011)

69. Reuter, J.S., Mathews, D.H.: RNAstructure: software for RNA secondary structure prediction and analysis. BMC Bioinform. 11(1), 1-9 (2010)

70. Ye, J., McGinnis, S., Madden, T.L.: BLAST: improvements for better sequence analysis. Nucleic Acids Res. 34 6-9 (2006)

71. Lu, R., Zhao, X., Li, J., Niu, P., Yang, B., Wu, H., Wang, W., Song, H., Huang, B., Zhu, N., et al.: Genomic characterisation and epidemiology of 2019 novel coronavirus: implications for virus origins and receptor binding. Lancet 395(10224), 565-574 (2020)

72. Rangan, R., Zheludev, I.N., Hagey, R.J., Pham, E.A., Wayment-Steele, H.K., Glenn, J.S., Das, R.: RNA genome conservation and secondary structure in SARS-CoV-2 and SARS-related viruses: a first look. RNA 26(8), 937-959 (2020)

73. Lan, T.C., Allan, M.F., Malsick, L., Khandwala, S., Nyeo, S.S., Bathe, M., Griffiths, A., Rouskin, S.: Structure of the full SARS-CoV-2 RNA genome in infected cells. bioRxiv (2020)

74. Schlick, T., Zhu, Q., Dey, A., Jain, S., Yan, S., Laederach, A.: To knot or not to knot: Multiple conformations of the SARS-CoV-2 frameshifting RNA element. J. Am. Chem. Soc. 143(30), 11404-11422 (2021)

75. Jones, C.P., Ferré-D'Amaré, A.R.: Crystal structure of the severe acute respiratory syndrome coronavirus 2 (SARS-CoV-2) frameshifting pseudoknot. RNA 28(2), 239-249 (2022)

76. Darty, K., Denise, A., Ponty, Y.: VARNA: Interactive drawing and editing of the RNA secondary structure. Bioinformatics 25(15), 1974 (2009)

77. Tsybulskyi, V., Mounir, M., Meyer, I.M.: R-chie: A web server and R package for visualizing cis and trans RNA-RNA, RNA-DNA and DNA-DNA interactions. Nucleic Acids Res. 48(18), 105-105 (2020)

\section{Figures}


Table 1: Structural similarity predictions for SARS-CoV, SARS-CoV-2, and MERS-CoV calculated using RNAz. Gaps in the alignment are represented as hyphen. Asterisks $(*)$ in bottom pseudoknot row correspond with known location of SARS-CoV-2 and MERS-CoV -1 PRF pseudoknot native structures.

\begin{tabular}{|c|c|}
\hline Virus & Secondary Structure Prediction \\
\hline SARS-CoV & 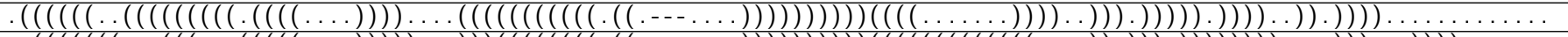 \\
\hline SARS-CoV-2 & $(\ldots)))))))))((((((((((((((\ldots)))))))))))).) \ldots \ldots))) \ldots)))) \ldots \ldots$ \\
\hline MERS-CoV & $(\ldots))()))))())) \ldots) \cdot(((((\ldots \ldots))))) \ldots)) \ldots))))))))(((\ldots$ \\
\hline Consensus &.$(((\ldots \ldots((((\ldots \ldots))))$. \\
\hline Pseudoknot & \\
\hline
\end{tabular}


Table 2: The predicted three most energetically favourable stems in SARS-CoV-2 -1 PRF stimulating pseudoknot reference sequence. These stems were used as structural guide for the Iterative HFold program to predict the SARS-CoV-2 secondary structure of the -1 PRF stimulating pseudoknot. First column provides stem ID and the third column lists free energy of the given stem. Input sequence provided in bottom row. See Table S2 for complete table with 18 stems.

\begin{tabular}{|c|c|c|}
\hline ID & Stem & Free Energy $(\mathrm{kcal} / \mathrm{mol})$ \\
\hline 1 & $\left(\left(\left(\left(\left(\left(\left(\left(\left(\left(\ldots_{\ldots} \ldots \ldots\right)\right)\right)\right)\right)\right)\right)\right)\right)\right)$ & -10.79 \\
\hline 2 & $\cdot((()((() \ldots))$ & -4.67 \\
\hline 3 &.$\cdot(((((\ldots))))$ & -3.77 \\
\hline Input sequence & GCGGUGUAAGUGCAGCCCGUCUUACACCGUGCGGCACAGGCACUAGUACUGAUGUCGUAUACAGGGCU & \\
\hline
\end{tabular}


Table 3: Predicted secondary structures for the SARS-CoV-2 -1 PRF stimulating pseudoknot based on the reference sequence. These structures are predicted by Iterative HFold given the input stems in Table S2 as structural constraints. Certain suboptimal structures (e.g. $6_{s}$ or $2_{s}$, cf. Figure 5 ) are reported because in these cases the structure has only slightly higher free energy than the minimum free energy structure predicted by Iterative HFold. Native structure is marked with an asterisk $\left(^{*}\right)$ in row 1 . As shown in rows 1,2 and 5 of the table, multiple initial stems can result in a single prediction for the -1 PRF stimulating pseudoknot. Input sequence provided in bottom row.

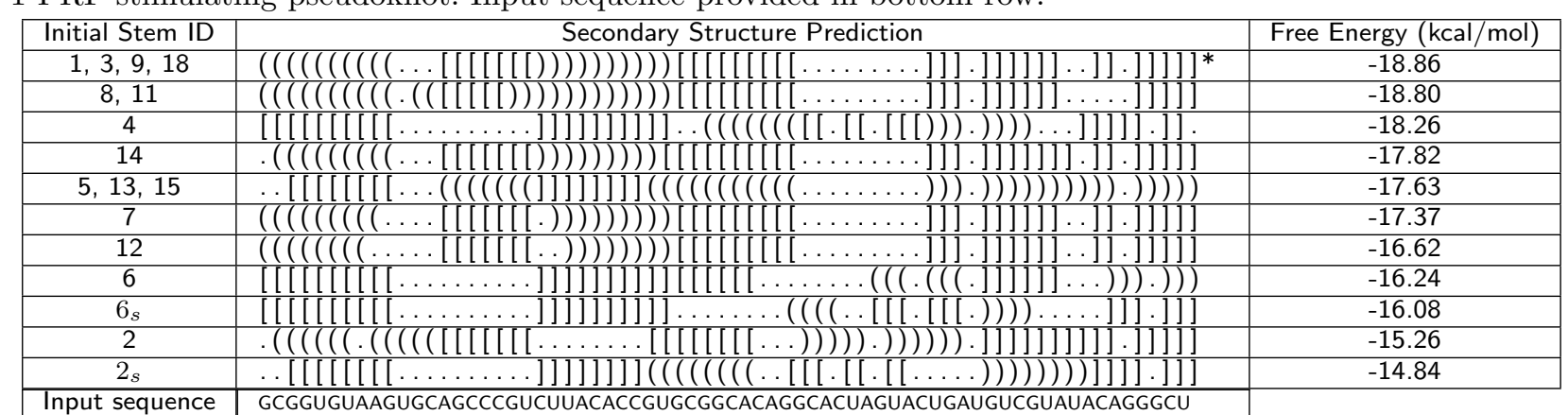


Table 4: Predicted most energetically favourable stems for SARS-CoV-2 -1 PRF stimulating pseudoknot mutated sequences. These stems were used as a structural guide for the Iterative HFold program to predict the SARS-CoV-2 secondary structure of the -1 PRF stimulating pseudoknot. Predictions in each row are based on the sequence with the mutation given in the first column. Second column provides stem ID and the fourth column provides free energy of the given stem. Stem IDs here correspond with stem free energy relative to the reference sequence initial stems. For example, in row one the stem has a free energy of -1.87 and is denoted by stem ID 13a. Relative to the stems predicted for the reference sequence (cf. Table 2), this stem has the thirteenth lowest free energy. Free energies were calculated using the HotKnots V2.0 energy parameters of Andronescu et al. [63]. Input sequence provided in bottom row of each mutation section, respective mutations italicized in red.

\begin{tabular}{|c|c|c|c|}
\hline Mutation & ID & Stem & Free Energy $(\mathrm{kcal} / \mathrm{mol})$ \\
\hline \multirow{2}{*}{$\mathrm{C} 13 \mathrm{U}$} & $13 a$ & $\ldots((((((\ldots \ldots))))))$ & -1.87 \\
\hline & & $\begin{array}{l}\text { GCGGUGUAAGUGUAGCCCGUCUUACACCGUGCGGCACAGGCACUAGUACUGAUGUCGUAUACAGGGCU } \\
\end{array}$ & \\
\hline \multirow{3}{*}{$\mathrm{U} 20 \mathrm{C}$} & $5 a$ & 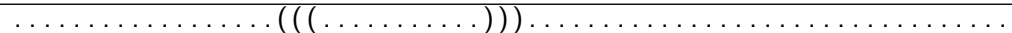 & -2.99 \\
\hline & $5 b$ & $\ldots((((\ldots \ldots \ldots \ldots)))) \ldots \ldots$ & -2.77 \\
\hline & & $\begin{array}{l}\text { GCGGUGUAAGUGCAGCCCGCCUUACACCGUGCGGCACAGGCACUAGUACUGAUGUCGUAUACAGGGCU } \\
\end{array}$ & \\
\hline \multirow{6}{*}{ G29U } & $2 a$ & 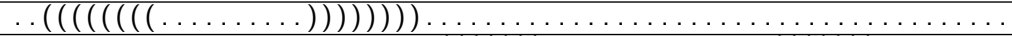 & -8.39 \\
\hline & $3 a$ & 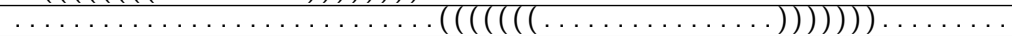 & -3.86 \\
\hline & $11 \mathrm{a}$ & $\ldots \ldots)((()($. & -2.13 \\
\hline & $13 \mathrm{~b}$ & 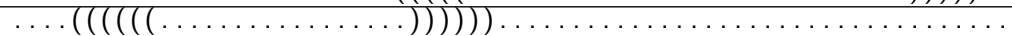 & -2.04 \\
\hline & $15 \mathrm{a}$ & 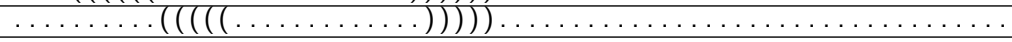 & -0.92 \\
\hline & & GCGGUGUAAGUGCAGCCCGUCUUACACCUUGCGGCACAGGCACUAGUACUGAUGUCGUAUACAGGGCU & \\
\hline \multirow{2}{*}{$\mathrm{C} 43 \mathrm{U}$} & $4 a$ & 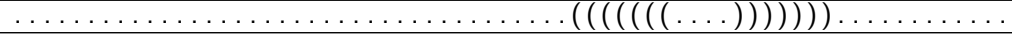 & -3.74 \\
\hline & & GCGGUGUAAGUGCAGCCCGUCUUACACCGUGCGGCACAGGCAUUAGUACUGAUGUCGUAUACAGGGCU & \\
\hline \multirow{3}{*}{ U47C } & $4 \mathrm{~b}$ & 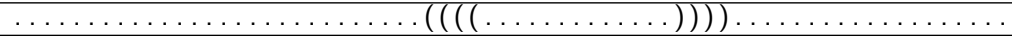 & -3.54 \\
\hline & $8 a$ & 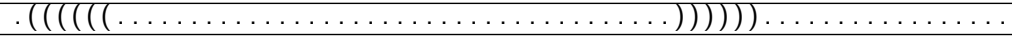 & -2.46 \\
\hline & & GCGGUGUAAGUGCAGCCCGUCUUACACCGUGCGGCACAGGCACUAGCACUGAUGUCGUAUACAGGGCU & \\
\hline \multirow{2}{*}{ U58C } & $18 \mathrm{a}$ & $\ldots((((\ldots))$ & -0.27 \\
\hline & & $\begin{array}{l}\text { GCGGUGUAAGUGCAGCCCGUCUUACACCGUGCGGCACAGGCACUAGUACUGAUGUCGCAUACAGGGCU } \\
\end{array}$ & \\
\hline \multirow{2}{*}{$\mathrm{C} 62 \mathrm{U}$} & $13 \mathrm{c}$ & 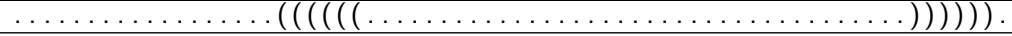 & -1.96 \\
\hline & & GGCACAGGCACUAGUACUGAUGUCGUAUAUAGGGCU & \\
\hline
\end{tabular}


Table 5: Predicted most energetically favourable stems for MERS-CoV -1 PRF stimulating pseudoknot reference sequence. These stems were used as a structural guide for the Iterative HFold program to predict the secondary structure of the -1 PRF stimulating pseudoknot. First column provides stem ID and the third column lists free energy of the given stem. Input sequence provided in bottom row. See Table S4 for complete table.

\begin{tabular}{|c|c|c|}
\hline ID & Stem & Free Energy $(\mathrm{kcal} / \mathrm{mol})$ \\
\hline 1 & $(((((((((((\ldots \ldots \ldots \ldots)))))))))))$ & -10.39 \\
\hline 2 &.$(((((\ldots \ldots))))) \ldots \ldots \ldots \ldots \ldots \ldots \ldots$ & -4.69 \\
\hline 3 & $.()))($. & -4.25 \\
\hline Tnput sequence & GGGGUUCUAUUGUAAAUGCCCGAAUAGAACCCUGUUCAAGUGGUUUGUCCACUGAUGUCGUCUUUAGGGCA & \\
\hline
\end{tabular}

\begin{tabular}{|l|l|}
\hline Input sequence & GGGGUUCUAUUGUAAAUGCCCGAAUAGAACCCUGUUCAAGUGGUUUGUCCACUGAUGUCGUCUUUAGGGCA \\
\hline
\end{tabular} 
Table 6: Predicted secondary structures for MERS-CoV -1 PRF stimulating pseudoknot based on the reference sequence. These structures are predicted by Iterative HFold given the input stems in Table 5 as structural constraints. Input sequence provided in bottom row. See Table S5 for complete table.

\begin{tabular}{|c|c|c|}
\hline Initial Stem ID & Secondary Structure Prediction & Free Energy $(\mathrm{kcal} / \mathrm{mol})$ \\
\hline 1,4 & $((((((((((((\ldots \ldots][[[[)))))))))).) \ldots[[[\cdot[[[[\ldots]]$ & -17.36 \\
\hline $2,3,8$ & ..)()) $)$ & -16.40 \\
\hline 12 & $((((\ldots))))$ & -16.17 \\
\hline Input sequence & GGGGUUCUAUUGUAAAUGCCCGAAUAGAACCCUGUUCAAGUGGUUUGUCCACUGAUGUCGUCUUUAGGGCA & \\
\hline
\end{tabular}


Table 7: ShapeKnots [28] secondary structure predictions for the SARS-CoV-2 -1 PRF stimulating pseudoknot based on the reference sequence. SHAPE dataset source for each prediction indicated in first column.

\begin{tabular}{|c|c|c|c|}
\hline Input Data & Secondary Structure Prediction & Free Energy $(\mathrm{kcal} / \mathrm{mol})$ \\
\hline Huston et al. $[30]$ & $\cdot(((((((((\ldots(([[[[[)))))))))))((((((((((\ldots \ldots \ldots))))))))).) \ldots]]]]]$ & -18.11 \\
\hline Manfredonia et al. $[29]$ & $\cdot(((((((((\ldots[[[[[[]))))))))((((((((((((\ldots \ldots)))))))))))]]]]]]$. & -17.25 \\
\hline Yang et al. [31] & $(((\ldots \ldots \ldots))((((((\ldots \ldots \ldots \ldots(((((((((((\ldots \ldots \ldots))))))))))) \ldots)))))$ & -10.39 \\
\hline Input sequence & GCGGUGUAAGUGCAGCCCGUCUUACACCGUGCGGCACAGGCACUAGUACUGAUGUCGUAUACAGGGCU & \\
\hline
\end{tabular}


Figure 1: SARS-CoV-2 Viral Genome. -1 PRF site marked by the red arrow. Normally, ribosome translates the complete ORF1a. Sometimes at the -1 PRF site, ribosome shifts from ORF1a to ORF1b, resulting in synthesis of fusion ORF1a/1b polypeptide.

Figure 2: Pseudoknot Diagram. (a) Hairpin loop with stem base pairs shown in red. H-type pseudoknot pairing shown in blue between bases in the loop and complementary downstream bases. (b) Representation of $2 \mathrm{a}$ in which arcs represent base pairs (arc diagram) for display of overlapping structures.

Figure 3: SARS-CoV-2 -1 PRF pseudoknot Native Structure. Stem 1 in red, Stem 2 in black, and pseudoknotted Stem 3 in blue. Visualization generated using VARNA [76].

Figure 4: Cross Validation Results. For each possible combination of the intercept and slope, the value within the grid represents the geometric mean of the sensitivity and positive predictive value over $30 \mathrm{RNA}$. Final result is an average of averages using a leave-one out scheme to avoid overweighting any one RNA. Optimal parameters identified by white box: intercept -0.5 , slope 1.4. Color indicates performance relative to optimal with darker green best and red the worst performance.

Figure 5: Initial Stem 2 Suboptimal Structure. Iterative HFold secondary structure predictions for SARS-CoV-2 -1 PRF stimulating pseudoknot with Stem 2 (in red) as constraint. Blue arcs correspond with the minimum free energy structure (cf. Table 3), black arcs represent a suboptimal structure with only slightly higher free energy. Visualization generated using R-chie web server [77]. 
Figure 6: Shapify Native-adjacent Pathways. Initial stems labeled on the left (e.g. S1 for initial Stem 1, cf. Table 2). Pathways from each initial stem lead to a secondary structure, either native or non-native (e.g. NN1 is the most stable nonnative structure, cf. Table S15). A black pathway from initial stem to secondary structure indicates this prediction was obtained for each of the three SHAPE datasets, respectively. A dark grey pathway indicates this prediction was obtained by using two of the SHAPE datasets, respectively, as constraint. A light grey pathway indicates the prediction was unique to using one SHAPE dataset as constraint. On the right, structures are presented in dot-bracket format, with the native structure in the first row. Differences from native structure are marked in bold, with brackets showing changes in paired bases, and asterisks representing unpaired bases that were paired in the native prediction.

Figure 7: Shapify Non-Native Pathways. Initial stems labeled on the left (cf. Table 2). Pathways from each initial stem lead to a non-native secondary structure. A black pathway indicates this prediction was obtained for each of the three SHAPE datasets, respectively. A dark grey pathway indicates this prediction was obtained by using two of the SHAPE datasets, respectively, as constraint. A light grey pathway indicates the prediction was unique to using one SHAPE dataset as constraint. On the right, structures are presented in dot-bracket format, native structure included in the first row for comparison. Differences from native structure are marked in bold, with brackets showing changes in paired bases, and asterisks representing unpaired bases that were paired in the native prediction.

Figure 8: SHAPE Dataset Analysis. (a) Comparison of -1 PRF sequence SHAPE reactivity dataset reported by Manfredonia et al. [29], Huston et al. [30], and Yang et al. [31]. Reactivity at or below 0.3 is considered to be low or non-reactive indicating the base is paired. (b) Each input dataset as displayed in Figure 8a was sampled with replacement and utilized as constraints for ShapeKnots [28] structure prediction 10,000 times. The mean and variance of the 10,000 predictions for each respective dataset are shown here, $y$-axis indicates the frequency each nucleotide is predicted as paired. Mean values of 1.0 convey the nucleotide was predicted as paired for all bootstrapped datasets, mean values of 0.0 indicates the nucleotide is predicted as unpaired for all bootstrapped datasets. (c) Bootstrap procedure as detailed was repeated with Shapify used for prediction. 
Figures

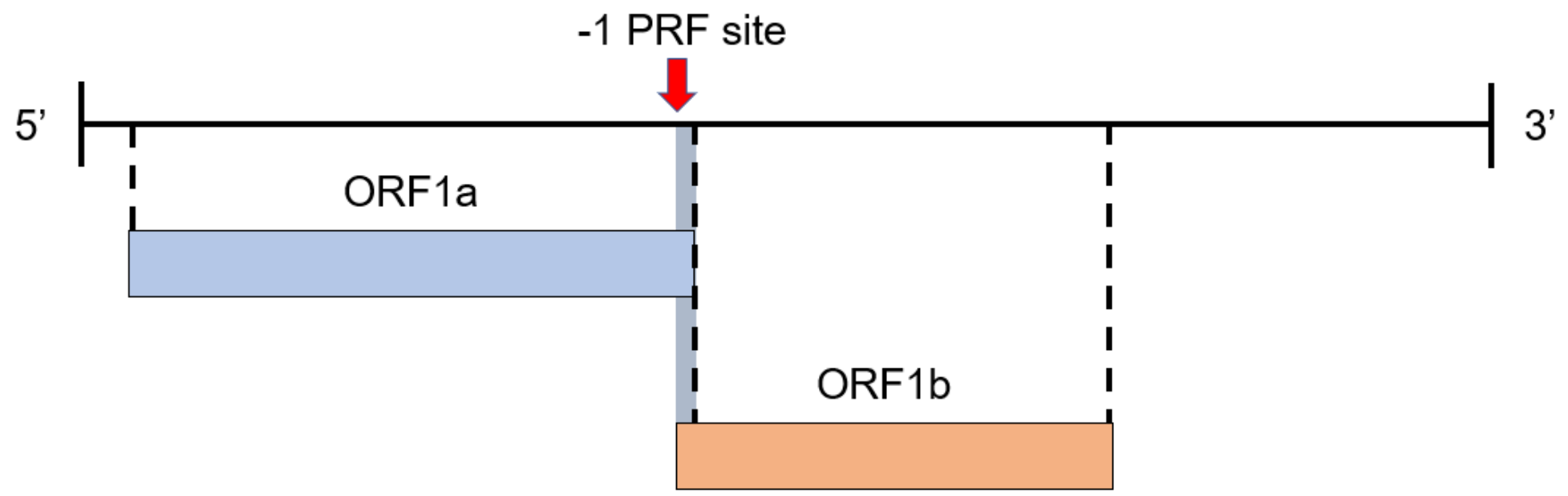

Figure 1

SARS-CoV-2 Viral Genome. - 1 PRF site marked by the red arrow. Normally, ribosome translates the complete ORF1a. Sometimes at the -1 PRF site, ribosome shifts from ORF1a to ORF1b, resulting in synthesis of fusion ORF1a/1b polypeptide. 


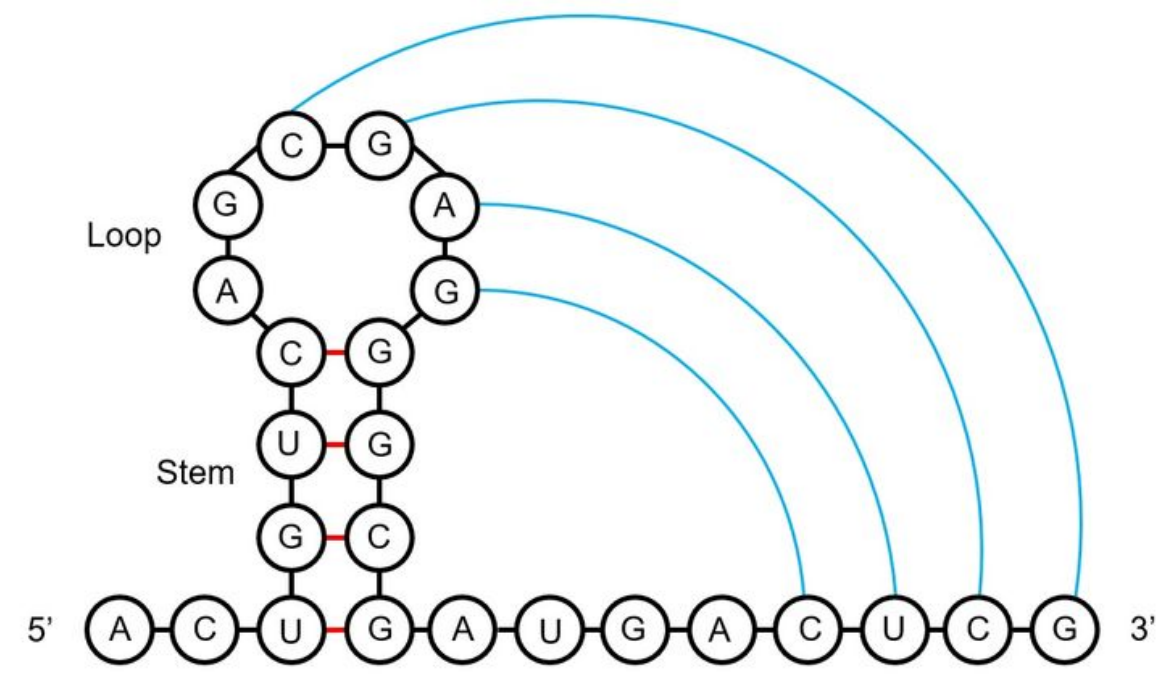

$2 a$

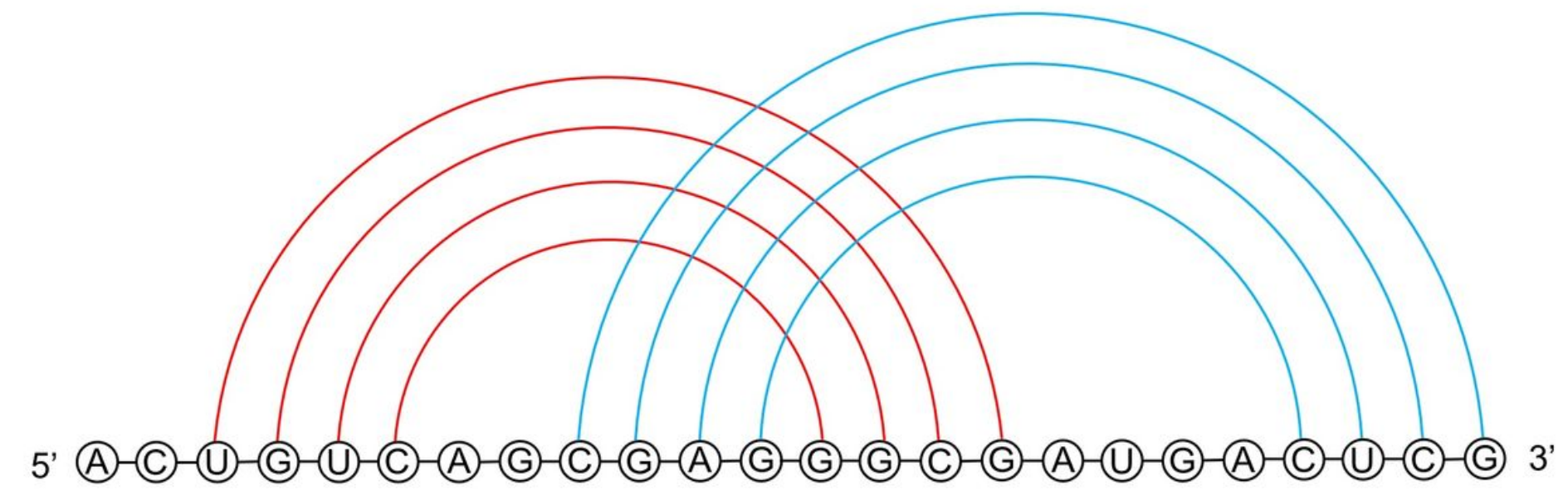

$2 b$

Figure 2

Pseudoknot Diagram. (a) Hairpin loop with stem base pairs shown in red. H-type pseudoknot pairing shown in blue between bases in the loop and complementary downstream bases. (b) Representation of $2 a$ in which arcs represent base pairs (arc diagram) for display of overlapping structures. 


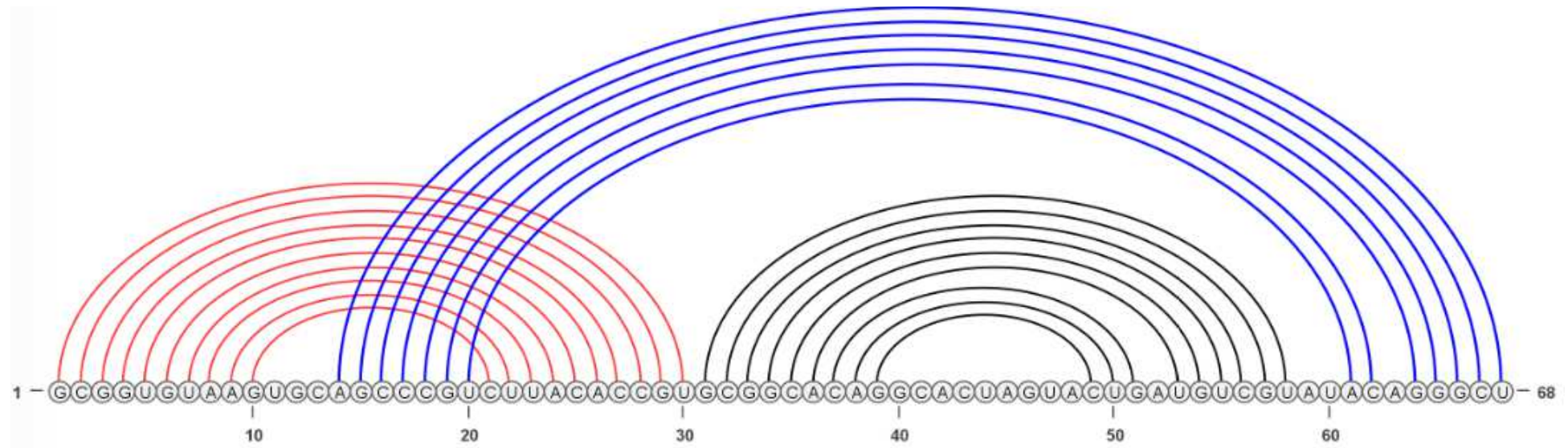

Figure 3

SARS-CoV-2 - 1 PRF pseudoknot Native Structure. Stem 1 in red, Stem 2 in black, and pseudoknotted Stem 3 in blue. Visualization generated using VARNA [76].

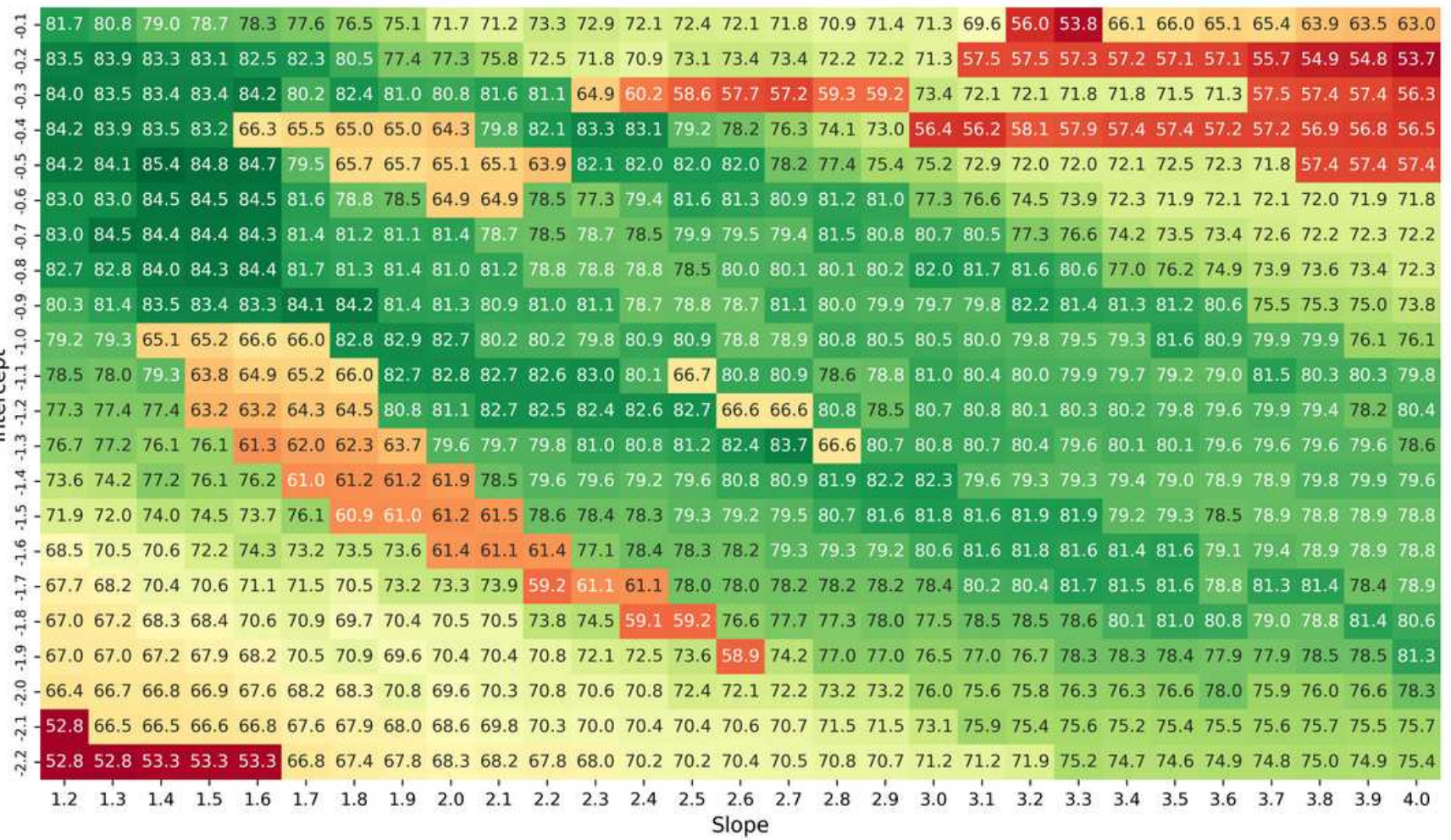

\section{Figure 4}

Cross Validation Results. For each possible combination of the inter-cept and slope, the value within the grid represents the geometric mean of the sensitivity and positive predictive value over 30 RNA. Final result is an average of averages using a leave-one out scheme to avoid overweighting any one RNA. Optimal parameters identified by white box: intercept $-0: 5$, slope 1:4. Color indicates performance relative to optimal with darker green best and red the worst performance. 


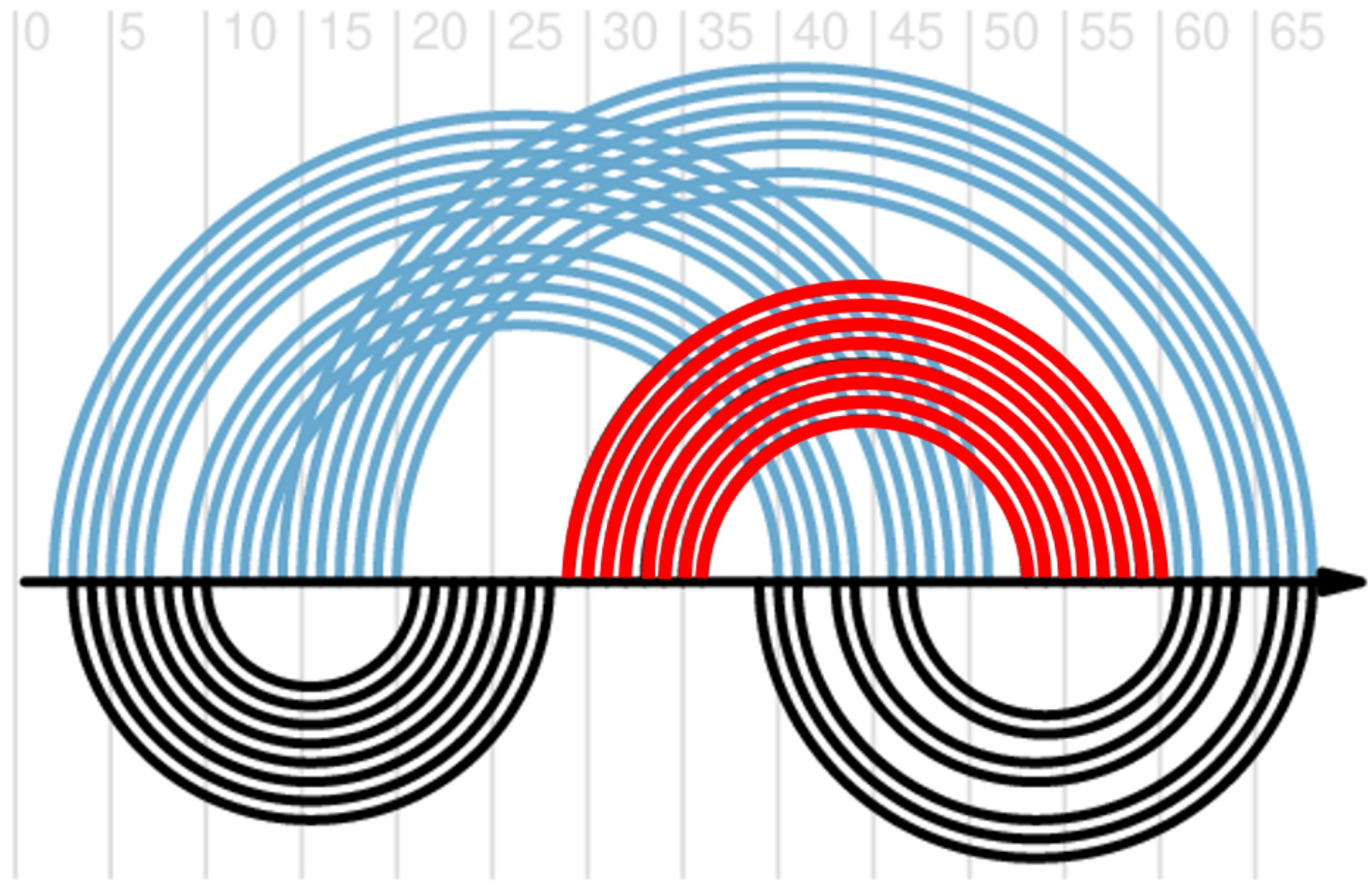

Figure 5

Initial Stem 2 Suboptimal Structure. Iterative HFold secondary structure predictions for SARS-CoV-2 -1 PRF stimulating pseudoknot with Stem 2 (in red) as constraint. Blue arcs correspond with the minimum free energy structure (cf. Table 3), black arcs represent a suboptimal structure with only slightly higher free energy. Visualization generated using R-chie web server [77]. 


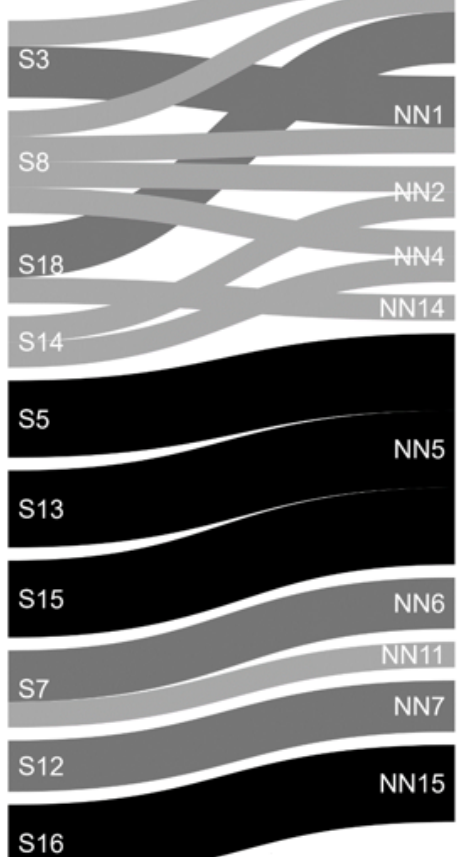

$((((((((()(([[[[[())))))))))))[[[[[[[[[\ldots \ldots \cdot]]] \cdot]]]]]] \ldots \star \star \cdot]]]]]$ $*\left(\left(\left(\left(\left(\left(\left(\left({ }^{2}[[[[[[[[())))))))) *[[[[[[[[\ldots \ldots \ldots]]]]]]].] \ldots\right]\right].\right]\right]\right]\right]\right]\right.$

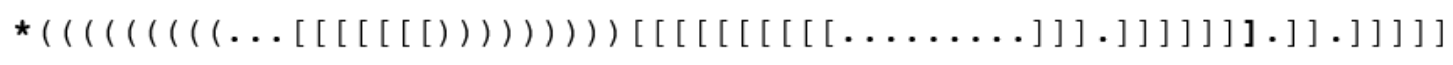

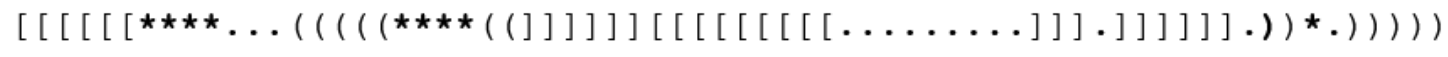

\section{Figure 6}

Shapify Native-adjacent Pathways. Initial stems labeled on the left (e.g. S1 for initial Stem 1, cf. Table 2). Pathways from each initial stem lead to a secondary structure, either native or non-native (e.g. NN1 is the most stable non-native structure, cf. Table S15). A black pathway from initial stem to secondary structure indicates this prediction was obtained for each of the three SHAPE datasets, respectively. A dark grey pathway indicates this prediction was obtained by using two of the SHAPE datasets, respectively, as constraint. A light grey pathway indicates the prediction was unique to using one SHAPE dataset as constraint. On the right, structures are presented in dot-bracket format, with the native structure in the rst row. Differences from native structure are marked in bold, with brackets showing changes in paired bases, and asterisks representing unpaired bases that were paired in the native prediction. 


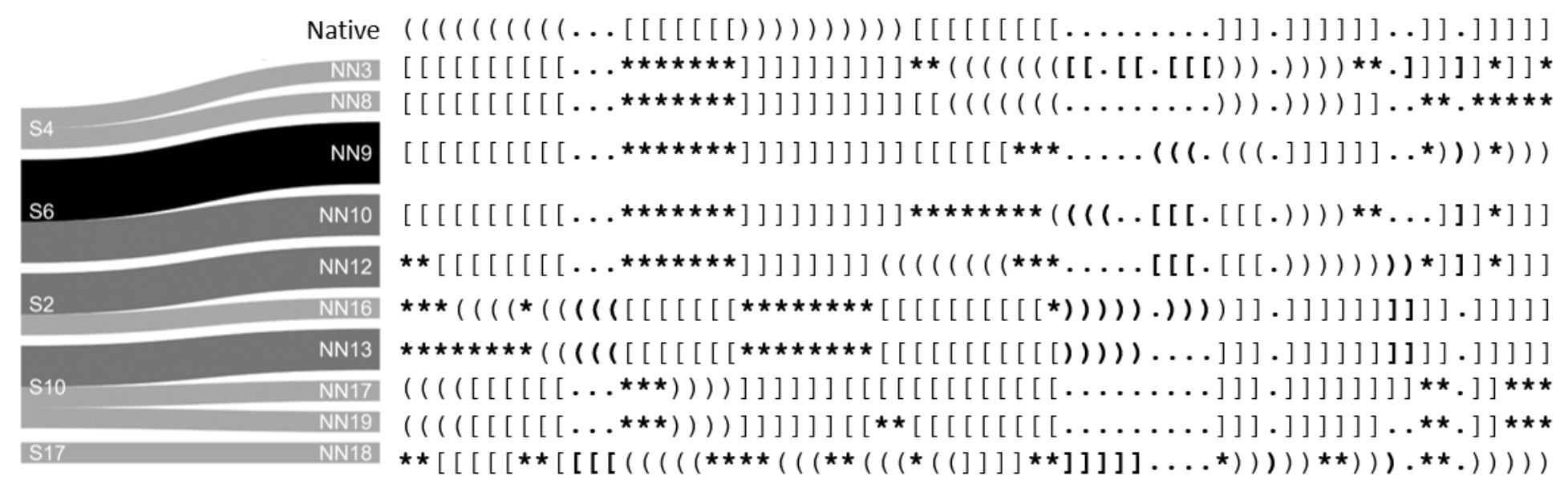

Figure 7

Shapify Non-Native Pathways. Initial stems labeled on the left (cf. Table 2). Pathways from each initial stem lead to a non-native secondary structure. A black pathway indicates this prediction was obtained for each of the three SHAPE datasets, respectively. A dark grey pathway indicates this prediction was obtained by using two of the SHAPE datasets, respectively, as constraint. A light grey pathway indicates the prediction was unique to using one SHAPE dataset as constraint. On the right, structures are presented in dot-bracket format, native structure included in the rst row for comparison. Differences from native structure are marked in bold, with brackets showing changes in paired bases, and asterisks representing unpaired bases that were paired in the native prediction. 


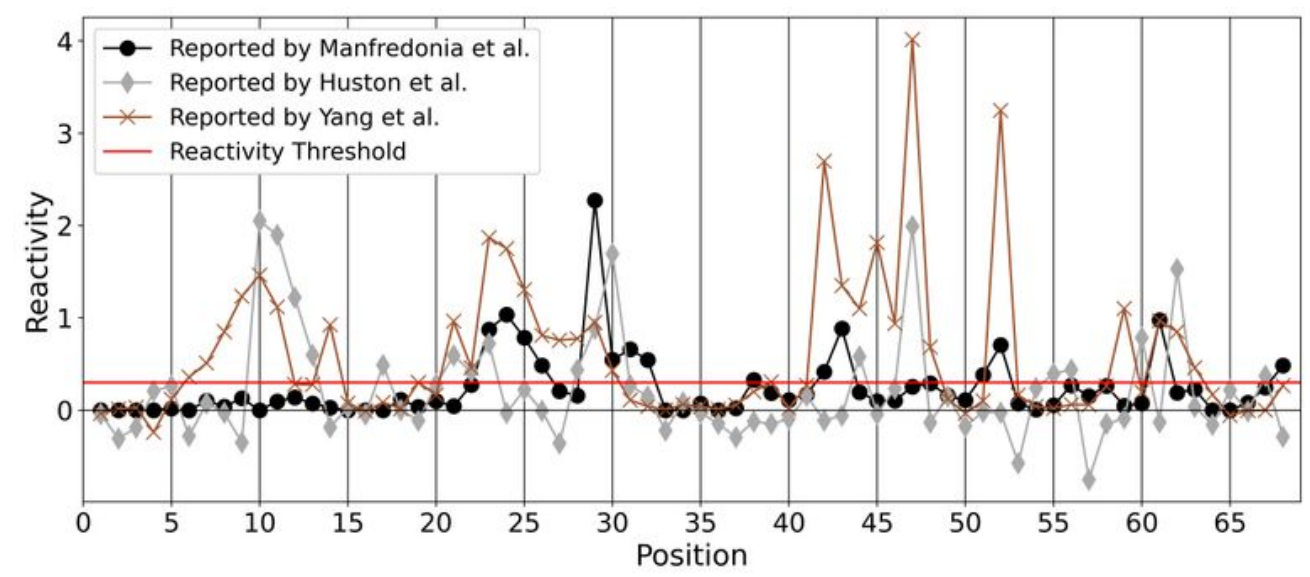

8a.

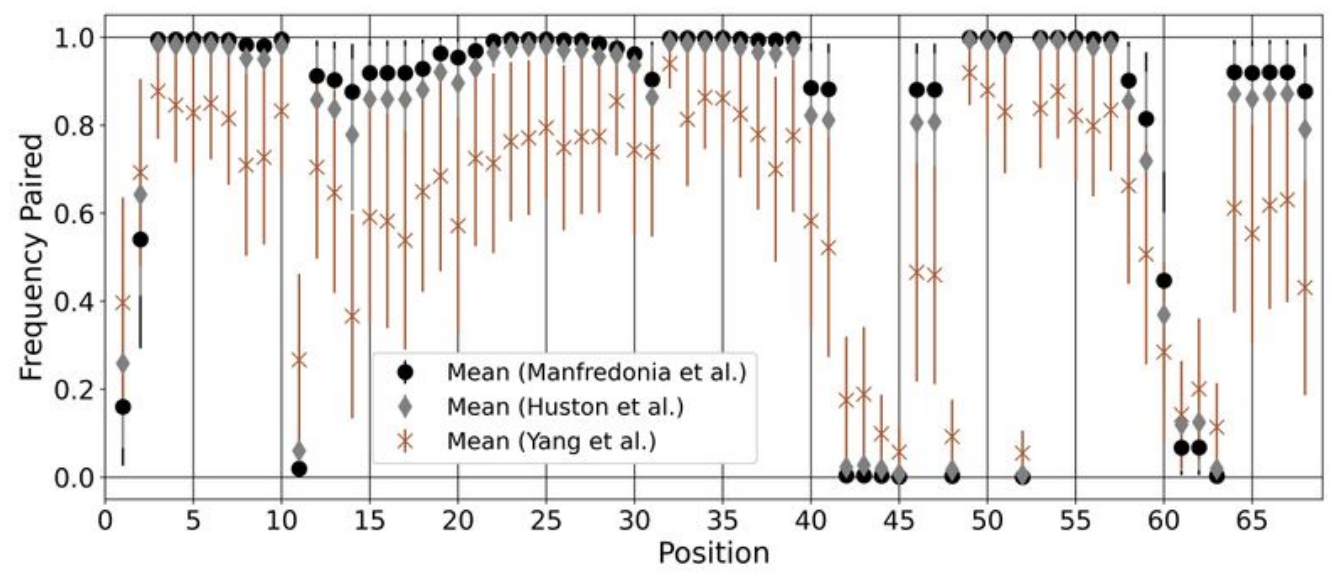

$8 b$.

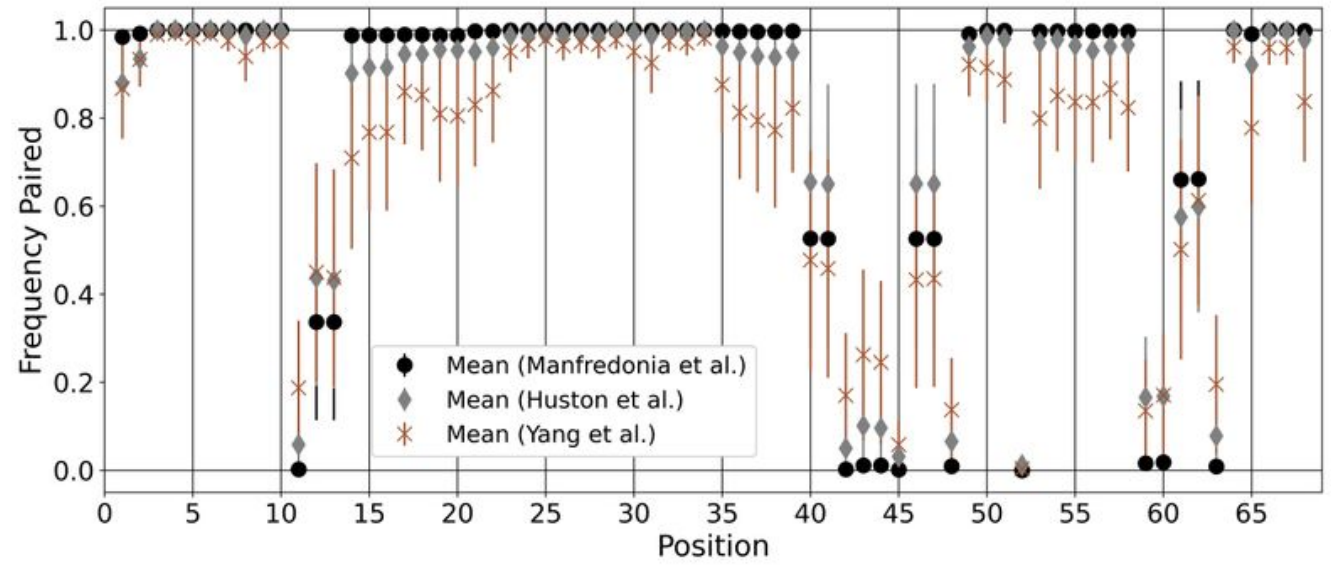

$8 c$.

Figure 8

SHAPE Dataset Analysis. (a) Comparison of -1 PRF sequence SHAPE reactivity dataset reported by Manfredonia et al. [29], Huston et al. [30], and Yang et al. [31]. Reactivity at or below 0.3 is considered to be low or non-reactive indicating the base is paired. (b) Each input dataset as displayed in Figure 8a was sampled with replacement and utilized as constraints for ShapeKnots [28] structure prediction 10; 000 times. The mean and variance of the 10; 000 predictions for each respective dataset are shown here, $y$ - 
axis indicates the frequency each nucleotide is predicted as paired. Mean values of 1:0 convey the nucleotide was predicted as paired for all bootstrapped datasets, mean values of 0:0 indicates the nucleotide is predicted as unpaired for all bootstrapped datasets. (c) Bootstrap procedure as detailed was repeated with Shapify used for prediction.

\section{Supplementary Files}

This is a list of supplementary files associated with this preprint. Click to download.

- TrinityFrameshiftingSupplemental.xIsx 